\title{
Three-body effective potential in general relativity at second post-Minkowskian order and resulting post-Newtonian contributions
}

\author{
Florian Loebbert@, ${ }^{*}$ Jan Plefka $\odot{ }^{\dagger}$ Canxin Shi, ${ }^{*}$ and Tianheng Wang ${ }^{\S}$ \\ Institut für Physik und IRIS Adlershof, Humboldt-Universiät zu Berlin, \\ Zum Großen Windkanal 6, 12489 Berlin, Germany
}

(Received 13 January 2021; accepted 11 February 2021; published 8 March 2021)

\begin{abstract}
We study the post-Minkowskian (PM) and post-Newtonian (PN) expansions of the gravitational threebody effective potential. At order 2PM a formal result is given in terms of a differential operator acting on the maximal generalized cut of the one-loop triangle integral. We compute the integral in all kinematic regions and show that the leading terms in the PN expansion are reproduced. We then perform the PN expansion unambiguously at the level of the integrand. Finding agreement with the $2 \mathrm{PN}$ three-body potential after integration, we explicitly present new $G^{2} v^{4}$-contributions at order 3PN and outline the generalization to $G^{2} v^{2 n}$. The integrals that represent the essential input for these results are obtained by applying the recent Yangian bootstrap directly to their $\epsilon$-expansion around three dimensions. The coordinate space Yangian generator that we employ to obtain these integrals can be understood as a special conformal symmetry in a dual momentum space.
\end{abstract}

DOI: $10.1103 /$ PhysRevD.103.064010

\section{INTRODUCTION}

The three-body problem in Newtonian gravity has been a source of inspiration in mathematics and physics since the time of Newton himself. Families of special solutions are known and tied to names such as Euler, Lagrange and Poincaré [1]. This system of nonintegrable differential equations poses a challenge to the theory of nonlinear systems and numerical approaches to date. They are of clear importance for celestial mechanics and space-flight, and have even been inspirational for science fiction [2]. In general relativity the problem is more challenging, as there are now genuine $\mathrm{N}$-body interactions going beyond the Newtonian 2-body potential. As observations indicate that many galaxies, including our own, contain supermassive black holes in their core, these $N$-body interactions might be important for the dynamics of multiple-star systems in their vicinity [3]. With the advent of gravitational wave astronomy [4-6] the gravitational radiation emitted by mergers of compact binaries is now observable. It is an interesting question whether genuine three-body systems,

\footnotetext{
*florian.loebbert@physik.hu-berlin.de

¡jan.plefka@hu-berlin.de

*canxin.shi@physik.hu-berlin.de

§tianheng.wang@physik.hu-berlin.de
}

Published by the American Physical Society under the terms of the Creative Commons Attribution 4.0 International license. Further distribution of this work must maintain attribution to the author(s) and the published article's title, journal citation, and DOI. Funded by SCOAP ${ }^{3}$. such as hierarchical systems where a black-hole binary is traversed by a third lighter compact object, will be observable in the future as well [7-11].

In the nonrelativistic (post-Newtonian) limit of general relativity the leading three-body interactions are due to Einstein, Infeld and Hoffmann [12,13] and arise from the effective potential [14]

$$
V_{3-\text { body }}^{1 \mathrm{PN}}=-\sum_{i=1}^{3} \sum_{\substack{j=1 \\ j \neq i}}^{3} \sum_{\substack{k=1 \\ k \neq i}}^{3} \frac{G^{2}}{2} \frac{m_{i} m_{j} m_{k}}{r_{i j} r_{i k}}
$$

with $r_{i j}$ denoting the spatial distance of the two massive bodies $m_{i}$ and $m_{j}, G$ is Newton's constant and we set $c=1$. In the nomenclature of the two-body problem this is the first post-Newtonian (1PN) contribution to the effective potential in which the velocity squared $v^{2}$ and the coupling term $\mathrm{Gm} / \mathrm{r}$ are of the same order due to the virial theorem. The velocity dependent $1 \mathrm{PN}$ terms contributing to the potential beyond (1) are pure two-body interactions of order $G v^{2}$. Numerical simulations of the relativistic threebody problem to date have mostly incorporated general relativity by restricting to the pure two-body $\mathrm{PN}$ terms to various orders [7,15-18], as the three-body interactions (1) and beyond are computationally costly, yet relevant $[3,8]$. In fact, a number of numerical studies incorporating the three-body interactions up to the presently known $2.5 \mathrm{PN}$ order exist [8,19-22] demonstrating their relevance for the dynamics. Simulations of three black holes in full numerical relativity [23-25] are challenging. 
The two-body conservative potential for spin-less compact binaries is known up to 4PN level for the potential [26-37], including parts of 5PN [38-40] and 6PN [41-46]. The situation for the $N$-body problem is considerably more open. For three bodies the effective potential is known to $2 \mathrm{PN}$ order $[8,19,47-49]$, generalizing (1) by three-body terms of order $G^{3} m^{4} / r^{3}$ as well as $v^{2} G^{2} m^{3} / r^{2}$ which entered the above-mentioned numerical studies $[8,19,20,22]$. The complexity of the three-body $2 \mathrm{PN}$ potential already increases considerably, cp. Eq. (52). For $N \geq 4$ the effective potential is in fact unknown at $2 \mathrm{PN}$ in an analytical form due to an unsolved two-loop spatial integral. The unintegrated $N$-body conservative potential at $2 \mathrm{PN}$ was presented in [50].

Turning to the weak gravitational field but arbitrary velocity limit-known as the post-Minkowskian (PM) limit, where one only expands in Newton's coupling but leaves the velocity inert - a lot of progress has been made on the two-body problem recently. Using methods of scattering amplitudes for perturbative quantum gravity, the $2 \mathrm{PM}[51,52]$ and $3 \mathrm{PM}[42,53,54]$ (including radiation reaction effects $[55,56])$ results for the effective potential have been established. A worldline effective field theory formalism for the PM expansion was recently formulated [57] and has now been successfully applied to order 3PM [58]. Earlier worldline-based PM calculations can be found in Refs. [59-63] for the conservative sector. The relation between the world-line quantum field theory and the scattering amplitude approach was recently clarified in [64]. Despite this progress, for the $N$-body problem nothing is known beyond 1PM order at which there are no genuine higher body interactions [61].

It is the aim of this paper to improve on this and to construct the 2PM effective potential in the three-body case (the essential 2PM formulae straightforwardly generalize to $N$ bodies). This in turn may be employed to determine all the velocity dependent contributions to the potential at order $G^{2}$ in the post-Newtonian expansion, i.e., the terms of order $v^{2 n} G^{2} m^{3} / r^{2}$. As the complexity of these contributions grows dramatically we shall explicitly provide only the so far unknown $v^{4} G^{2} m^{3} / r^{2}$ terms which contribute to the 3PN terms in the potential in Sec. VII. The general tools to determine the higher velocity terms will be provided.

We employ the PM worldline effective quantum field theory formalism based on $[57,64]$, generalizing the nonrelativistic (PN) effective field theory approach of [65] to general relativity. The three-body $2 \mathrm{PM}$ potential essentially follows from a single Feynman diagram connecting the three-graviton vertices with the world-lines resulting in a one-loop three-point integral with coordinate space Green's functions [59]. This integral features a Yangian level-one symmetry $[66,67]$ and is related to a generalized cut of the four-point box integral, which has recently been obtained from Yangian bootstrap [68,69]. Generalizing the calculation of [59], we explicitly show that our three-point integral is indeed proportional to one of the four Yangian invariants found in [68]. We then demonstrate that the PN expansion is most efficiently performed at the integrand level, which results in a family of three-point integrals in three dimensions with half integer propagator powers. Again, this family of divergent integrals is invariant under a Yangian level-one generator, which allows to bootstrap their expansion in the dimensional regularization parameter $\epsilon$. This level-one symmetry can alternatively be interpreted as a special conformal symmetry in a dual momentum space, cf. [70-72] for the connection between the two symmetries. We explicitly perform the PN expansion to NNLO yielding the previously unknown $v^{4} G^{2} m^{3} / r^{2}$ terms at the 3PN level and illustrate the generalization to $v^{2 n} G^{2} m^{3} / r^{2}$.

This paper is organized as follows: after a general discussion of the worldline effective field theory in the Polyakov formulation in Sec. II we construct the 2PM potential in Sec. III. The computation of the emerging three-point key integral in various kinematical regions is relegated to Appendix A. Section IV discusses the 1PN limit of the 2PM potential recovering the Einstein-Infeld-Hofmann Lagrangian. In Sec. V we lay out our general approach to integrate the $2 \mathrm{PM}$ potential in the nonrelativistic $\mathrm{PN}$ expansion at the level of the integrand making use of a level-one Yangian symmetry for the emerging master integrals. As concrete applications of this procedure we then recover the known 2PN three-body potential up to the static term in Sec. VI, and in Sec. VII provide all three-body terms at the 3PN order that scale quartically in velocities and show that they reproduce the known results in the twobody limit.

\section{EFFECTIVE FIELD THEORY}

Consider three massive spinless point particles coupled to Einstein gravity via the action

$$
S=S_{\mathrm{EH}}+S_{\mathrm{gf}}+S_{\mathrm{pp}}
$$

Here we have defined

$$
\begin{aligned}
S_{\mathrm{EH}} & =-\frac{2}{\kappa^{2}} \int d^{4} x \sqrt{-g} R+(\mathrm{GHY} \text { term }) \\
& =-\frac{2}{\kappa^{2}} \int d^{4} x \sqrt{-g}\left[g^{\mu \nu}\left(\Gamma_{\mu \lambda}^{\rho} \Gamma_{\nu \rho}^{\lambda}-\Gamma_{\mu \nu}^{\rho} \Gamma_{\rho \lambda}^{\lambda}\right)\right],
\end{aligned}
$$

with $\kappa^{2}=32 \pi G$, the gravitational coupling and a GibbonsHawking-York (GHY) boundary term [73,74]. For the point particles we start out with the action

$$
S_{\mathrm{pp}}^{\prime}=-\sum_{i} m_{i} \int d \tau_{i} \sqrt{g_{\mu \nu}\left(x\left(\tau_{i}\right)\right) u_{i}^{\mu}\left(\tau_{i}\right) u_{i}^{\nu}\left(\tau_{i}\right)},
$$

with the 4-velocities $u_{i}^{\mu}=\frac{d x_{i}^{\mu}}{d \tau_{i}}$ integrated along their worldlines. It turns out to be more advantageous to work with the 
Polyakov formulation of the point-particle action. Upon introducing the einbein $e_{i}=e\left(x\left(\tau_{i}\right)\right)$ this action reads

$$
S_{\mathrm{pp}}=-\sum_{i=1}^{3} \frac{m_{i}}{2} \int d \tau_{i} e_{i}\left(g_{\mu \nu} u_{i}^{\mu}\left(\tau_{i}\right) u_{i}^{\nu}\left(\tau_{i}\right)+\frac{1}{e_{i}^{2}}\right),
$$

which preserves reparametrization invariance by the transformation rule for $e_{i}$. Solving the algebraic equations of motion for the inverse einbein yields $e_{i}^{-1}=$ $\sqrt{g_{\mu \nu} u_{i}^{\mu}\left(\tau_{i}\right) u_{i}^{\nu}\left(\tau_{i}\right)}$ and plugging this back into the action recovers the original action (4).

In the weak field expansion of the metric we take $g_{\mu \nu}=\eta_{\mu \nu}+\kappa h_{\mu \nu}$, using the mostly minus convention. We choose the standard de Donder gauge fixing term $S_{\text {gf }}=\int d^{4} x f_{\mu} f^{\mu}$ with $f^{\mu}=\partial^{\nu} h^{\mu}{ }_{\nu}-\frac{1}{2} \partial^{\mu} h^{\nu}{ }_{\nu}$. This yields the graviton Feynman propagator

$$
\approx=\frac{i}{2} \frac{P^{\mu \nu \rho \sigma}}{k^{2}+i \varepsilon}
$$

with $P^{\mu \nu \rho \sigma}=\eta^{\mu \rho} \eta^{\nu \sigma}+\eta^{\mu \sigma} \eta^{\nu \rho}-\eta^{\mu \nu} \eta^{\rho \sigma}$. The advantage of the Polyakov formulation (5) is that it only gives rise to a single graviton worldline interaction:

$$
\approx=-i \kappa e(\tau) u^{\mu}(\tau) u^{\nu}(\tau)
$$

In the bulk we will only need the three-graviton vertex, which may be found, e.g., in [75]. An important aspect in the construction of the classical effective action is the causality structure of the propagator as was recently stressed in [55]. The Fourier transform to coordinate space of the graviton Feynman propagator reads $\left(x_{i j}^{\mu}=x_{i}^{\mu}-x_{j}^{\mu}\right)$

$$
\begin{aligned}
\bar{D}_{i j} & =\int \frac{\mathrm{d}^{4} k}{(2 \pi)^{4}} \frac{1}{k^{2}+i \varepsilon} e^{i k \cdot x_{i j}} \\
& =\frac{1}{4 \pi^{2}} \frac{i}{x_{i j}^{2}-i \varepsilon}=-\frac{1}{4 \pi} \delta\left(x_{i j}^{2}\right)+\frac{i}{4 \pi^{2} x_{i j}^{2}},
\end{aligned}
$$

where the famous distributional identity

$$
\lim _{\epsilon \rightarrow 0^{+}} \frac{1}{y \pm i \epsilon}=\mathrm{pv} \frac{1}{y} \mp i \pi \delta(y)
$$

was used in the last step, thereby dropping the principal value label. In order to construct the classical, conservative action for PM gravity, one should restrict to the real part $D_{i j}$ defined as:

$$
D_{i j}=\operatorname{Re}\left(\bar{D}_{i j}\right)=-\frac{1}{4 \pi} \delta\left(x_{i j}^{2}\right) .
$$

This propagator obeys the Green's function identity

$$
\square D_{i j}=-\delta^{(4)}\left(x_{i j}\right)
$$

It may also be expressed as

$$
\delta\left(x^{2}\right)=\frac{\delta(c t-r)}{2 r}+\frac{\delta(c t+r)}{2 r},
$$

where $r=|\mathbf{x}|$, making manifest that the sum of the retarded and advanced propagator, i.e., the time symmetric propagator, is the real part of the Feynman propagator. The conservative effective action $S_{\text {eff }}$ may then be obtained upon integrating out the graviton fluctuations.

\section{2PM POTENTIAL}

Up to order 2PM the effective action is expanded as

$$
S_{\text {eff }}=S^{\text {free }}+\kappa^{2} S^{1 \mathrm{PM}}+\kappa^{4} S^{2 \mathrm{PM}}+\mathcal{O}\left(\kappa^{6}\right),
$$

where the free contribution takes the form of (5) with $g_{\mu \nu}$ replaced by $\eta_{\mu \nu}$. Using the Feynman rule (7), it is straightforward to compute the 1PM order. It follows from a single graviton exchange between each pair of point masses

$$
\begin{aligned}
\kappa^{2} S^{1 \mathrm{PM}} & =\sum_{i} \sum_{j \neq i} \underset{i}{\approx} \\
& =\sum_{i} \sum_{j \neq i} \int \mathrm{d} \hat{\tau}_{i} \mathrm{~d} \hat{\tau}_{j} \frac{\kappa^{2} m_{i} m_{j}}{32 \pi}\left[u_{i j}^{2}-\frac{1}{2} u_{i}^{2} u_{j}^{2}\right] \delta\left(x_{i j}^{2}\right),
\end{aligned}
$$

with $d \hat{\tau}_{i}:=e_{i} d \tau_{i}$, and $u_{j k}:=u_{j} \cdot u_{k}$. There are no threebody interactions at this order. Moving on to 2PM, we find the first genuine three-body interaction in the theory, which arises from a single Feynman diagram. In coordinate space it reads

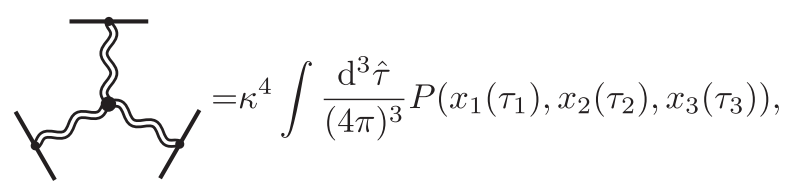

where we have defined $\mathrm{d}^{3} \hat{\tau}=\mathrm{d} \hat{\tau}_{1} \mathrm{~d} \hat{\tau}_{2} \mathrm{~d} \hat{\tau}_{3}$ as well as

$$
\begin{aligned}
8 & \left(m_{1} m_{2} m_{3}\right)^{-1} P\left(x_{i}\left(\tau_{i}\right)\right) \\
:= & \pi\left(4 u_{12}^{2} u_{3}^{2}-4 u_{12} u_{13} u_{23}-u_{1}^{2} u_{2}^{2} u_{3}^{2}\right) \delta\left(x_{12}^{2}\right) \delta\left(x_{13}^{2}\right) \\
& +\left(u_{12}^{2} u_{3}^{\mu} u_{3}^{\nu}-\frac{1}{2} u_{1}^{2} u_{2}^{2} u_{3}^{\mu} u_{3}^{\nu}+2 u_{13} u_{23} u_{2}^{\mu} u_{1}^{\nu}\right) \partial_{x_{1}, \mu} \partial_{x_{2}, \nu} I_{3 \delta} \\
& + \text { (cyclic) },
\end{aligned}
$$

with the integral $I_{3 \delta}$ further discussed below. Note that we have discarded all terms proportional to $u_{i} \cdot \partial_{x_{i}}$, which can be written as derivatives $d / d \tau_{i}$. Modulo integration by 
parts, the $\tau_{i}$-derivatives act on the $u_{i}$ and the einbein $e_{j}$, which also solely depends on the $u_{j}$ after solving the equations of motion, see (22). In the end, the $\tau_{i}$-derivatives yield terms involving accelerations, which, by proper field redefinition of $x_{i}$, can be replaced by lower order equations of motion that lift these terms to the next order in $\kappa^{2}$, cf. [76]. Note that we will employ this mechanism at several points of the paper.

To complete the three-body action we need to include the two-body interactions at 2PM. These can be obtained from (15) by identifying two of the three world-lines and multiplying with a symmetry factor $1 / 2$. The full $2 \mathrm{PM}$ three-body action thus becomes

$$
S^{2 \mathrm{PM}}=\frac{1}{6} \int \frac{\mathrm{d}^{3} \hat{\tau}}{(4 \pi)^{3}} \sum_{i, j, k}^{\prime} P\left(x_{i}\left(\tau_{1}\right), x_{j}\left(\tau_{2}\right), x_{k}\left(\tau_{3}\right)\right),
$$

where the sum $\sum_{i, j, k}^{\prime}$ runs over $i, j, k=1,2,3$ but excludes $i=j=k$. Moreover, propagators that have both ends on the same worldline vanish in dimensional regularization. We note that in fact this becomes the $N$-body 2PM action if we allow $i, j, k$ to run from 1 to $N$.

The central ingredient in the above formula (16) for the three-body contribution to the effective potential is the integral

$$
I_{3 \delta}:=\int \mathrm{d}^{4} x_{0} \delta\left(x_{01}^{2}\right) \delta\left(x_{02}^{2}\right) \delta\left(x_{03}^{2}\right)=x_{1}
$$

which is interesting for various reasons. In the present paper it arises as the one-loop three-point integral in coordinate space (black solid diagram). Alternatively, we can interpret it as the generalized maximal cut of the momentum space triangle integral where all propagators are put on shell (green dashed diagram), expressed in terms of region momenta $x_{j}$, which map to the dual momenta $R_{j}$ via

$$
R_{j}^{\mu}:=x_{j+1}^{\mu}-x_{j-1}^{\mu} .
$$

Moreover, $I_{3 \delta}$ is related to a generalized cut of the fourpoint (box) integral, in the limit where one point is sent to infinity. The box integral is invariant under a Yangian algebra, an extension of its well known conformal symmetry $[66,77]$. As such, in the region $R_{j}^{2}<0$ the integral is given by the minimal transcendentally solution of the Yangian constraints found in [68] (modulo a piecewise constant):

$$
I_{3 \delta}=\frac{C}{\sigma}, \quad \sigma^{2}:=\left(R_{2} \cdot R_{3}\right)^{2}-R_{2}^{2} R_{3}^{2} .
$$

Note that due to $R_{1}+R_{2}+R_{3}=0$ this representation is not unique and one may pick any two $R_{i}$ 's to define $\sigma^{2}$.

To obtain $I_{3 \delta}$, it is useful to generalize the steps of Westpfahl [59], who evaluated the integral for the retarded propagator. This generalization performed in Appendix A shows that the value of the integral depends on the sign of $\sigma^{2}$. In fact, for $R_{j}^{2}<0$ with $j=1,2,3$ the expression (20) can be compared with the result of [59] which shows that $C\left(\sigma^{2}>0, R_{j}^{2}<0\right)=\pi / 4$ in the above expression. However, more care is needed to obtain $C$ for generic kinematics. The explicit calculation given in Appendix A shows that for $\sigma^{2}>0$ we have

$$
I_{3 \delta}=\frac{\pi}{4 \sigma} \Theta\left(-R_{1}^{2} R_{2}^{2} R_{3}^{2}\right) .
$$

Here $\Theta$ denotes the Heaviside-function as defined in (A7). For $\sigma^{2}<0$ the integral diverges and for $\sigma^{2}=0$ it is proportional to $\sum_{i} \delta\left(R_{j}^{2}\right)$, see Appendix A.

\section{THE 1PN EXPANSION}

In this section we want to provide a first test of the above expression for the full $2 \mathrm{PM}$ effective action against known results for the three-body potential at $1 \mathrm{PN}$ order. For this we first solve the equation of motion $\delta S / \delta e_{i}=0$ for $e_{i}$ perturbatively up to order $\kappa^{2}$ :

$e_{i}=\frac{1}{\sqrt{u_{i}^{2}}}+\sum_{j \neq i} \int \mathrm{d} \tau_{j} \frac{\kappa^{2} m_{j}}{16 \pi \sqrt{u_{i}^{6} u_{j}^{2}}}\left(u_{i j}^{2}-\frac{1}{2} u_{i}^{2} u_{j}^{2}\right)+\mathcal{O}\left(\kappa^{4}\right)$.

Plugging this solution back into (13) and expanding to order $\kappa^{4}$ yields the 2PM effective action free of the einbein. We then consider its nonrelativistic limit, choosing the convenient gauge $\tau_{i}=t_{i}$. Reintroducing the speed of light $c$ such that

$u_{i}^{\mu}=\left(1, \frac{\mathbf{v}_{i}}{c}\right), \quad \frac{\partial}{\partial x_{i}^{\mu}}=\left(\frac{\partial}{c \partial t_{i}}, \frac{\partial}{\partial \mathbf{x}_{i}}\right), \quad \kappa \rightarrow \frac{\kappa}{c}$,

we see that in $P\left(x_{i}\right)$ of (16) only the second line contributes at leading order in $c^{-1}$ :

$\sum_{i, j, k}^{\prime} P\left(x_{i}\right)=-\frac{3 \pi m_{1} m_{2} m_{3}}{8} \sum_{i} \sum_{\substack{j \neq i \\ k \neq i}} \delta\left(x_{i j}^{2}\right) \delta\left(x_{i k}^{2}\right)+\mathcal{O}\left(c^{-2}\right)$.

Note that we have rewritten the sum by discarding propagators with both ends on the same worldline. Using the nonrelativistic expansion of the propagator (10) 


$$
\begin{aligned}
\delta\left(x_{i j}^{2}\right)= & \frac{\delta\left(t_{i}-t_{j}\right)}{r_{i j}}-\frac{r_{i j}}{2 c^{2}} \partial_{t_{i}} \partial_{t_{j}} \delta\left(t_{i}-t_{j}\right) \\
& +\frac{r_{i j}^{3}}{24 c^{4}} \partial_{t_{i}}^{2} \partial_{t_{j}}^{2} \delta\left(t_{i}-t_{j}\right)+\mathcal{O}\left(c^{-4}\right),
\end{aligned}
$$

where $r_{i j}=\left|\mathbf{r}_{i j}\right|$ with $\mathbf{r}_{i j}=\mathbf{x}_{i}-\mathbf{x}_{j}$, yields a localized time integration in the effective action (17). After some rearrangements, we find the 1PN three-body effective action ${ }^{1}$

$$
\begin{aligned}
S= & \sum_{i} \int d t\left[-m_{i}+\frac{1}{c^{2}}\left(\frac{m_{i} \mathbf{v}_{i}^{2}}{2}+\sum_{j \neq i} \frac{G m_{i} m_{j}}{2 r_{i j}}\right)\right. \\
& +\frac{1}{c^{4}}\left(\frac{m_{i} \mathbf{v}_{i}^{4}}{8}+\sum_{j \neq i} \frac{G m_{i} m_{j}}{4 r_{i j}}\left(6 \mathbf{v}_{i}^{2}-\left(\mathbf{n}_{i j} \cdot \mathbf{v}_{i}\right)\left(\mathbf{n}_{i j} \cdot \mathbf{v}_{j}\right)\right.\right. \\
& \left.\left.\left.-7 \mathbf{v}_{i} \cdot \mathbf{v}_{j}\right)-\sum_{j \neq i} \sum_{k \neq i} \frac{G^{2} m_{i} m_{j} m_{k}}{2 r_{i j} r_{i k}}\right)\right]
\end{aligned}
$$

where we abbreviate $\mathbf{n}_{i j}:=\mathbf{r}_{i j} / r_{i j}$ and $G=\kappa^{2} / 32 \pi$. This result agrees with the well known 1PN expression [14].

\section{POST-NEWTONIAN EXPANSION AND INTEGRAL BOOTSTRAP}

The 1PN expansion obtained in the previous section merely tests the second line of the three-body contribution (16) to the effective potential. In order to obtain the expansion at $2 \mathrm{PN}$ order, also the third line in (16) has to be taken into account. This includes second derivatives of the three-delta integral, $\partial_{j}^{\mu} \partial_{k}^{\nu} I_{3 \delta}$, cf. the $\Theta$-function in (21). As outlined in detail in Appendix B, taking these derivatives leads to lengthy expressions in terms of delta functions and their derivatives which are hard to control. In fact, it is simpler to perform the nonrelativistic expansion directly on the level of the integrand of $I_{3 \delta}$ as we will demonstrate in the following. For convenience of the reader we briefly summarize the below strategy: First, we will show that expanding the integrand of $I_{3 \delta}$ leads to the family of key integrals given in (31). We will then use the Yangian level-one symmetry of these integrals, i.e., invariance under the differential operator (33), to obtain the differential equations (36). Finally, we explicitly demonstrate how these equations are solved in the form of (39), which results in the expressions for the dimensionally regularized integrals that enter into the effective potential. To start we consider the nonrelativistic expansion of the propagator of (8), generalized to $D$ spatial dimensions in the so-called potential region $\omega:=k^{0} \ll|\mathbf{k}|$, writing

$$
\frac{1}{k^{2}}=\frac{1}{\omega^{2}-\mathbf{k}^{2}}=-\sum_{\alpha=1}^{\infty} \frac{\omega^{2 \alpha-2}}{\left(\mathbf{k}^{2}\right)^{\alpha}} .
$$

\footnotetext{
${ }^{1}$ Note that in the GR literature the PN action is typically rescaled by a factor of $c^{2}$.
}

Inserting this expansion into the Fourier transformed expression for the time-symmetric propagator yields the common $\mathrm{PN}$-expanded propagator

$$
\delta\left(x_{0 i}^{2}\right)=4 \pi \int \frac{\mathrm{d}^{D} k}{(2 \pi)^{D}} e^{i \mathbf{k} \cdot \mathbf{x}_{0 i}} \sum_{\alpha=0}^{\infty} \frac{(-1)^{\alpha} \partial_{t_{i}}^{2 \alpha} \delta\left(t_{0 i}\right)}{c^{2 \alpha}\left(\mathbf{k}^{2}\right)^{\alpha+1}},
$$

having performed the energy $(\omega)$ integral. Hence, with the expression for the $D$-dimensional Fourier transform of the momentum space propagator,

$$
\int \frac{\mathrm{d}^{D} k}{(2 \pi)^{D}} \frac{e^{i \mathbf{k} \cdot \mathbf{x}}}{\left(\mathbf{k}^{2}\right)^{\alpha}}=\frac{1}{4^{\alpha} \pi^{D / 2}} \frac{\Gamma_{D / 2-\alpha}}{\Gamma_{\alpha}} r^{2 \alpha-D},
$$

we can write the key integral $I_{3 \delta}$ in the PN-expansion for general spatial $D$ as

$$
\begin{aligned}
I_{3 \delta}= & \sum_{\alpha, \beta, \gamma=0}^{\infty} \frac{(-1)^{\alpha+\beta+\gamma}}{(2 c)^{2(\alpha+\beta+\gamma)} \pi^{3(D / 2-1)}} \frac{\Gamma_{\hat{\alpha}} \Gamma_{\hat{\beta}} \Gamma_{\hat{\gamma}}}{\Gamma_{\alpha+1} \Gamma_{\beta+1} \Gamma_{\gamma+1}} \\
& \times \int \mathrm{d} t_{0} \partial_{t_{1}}^{2 \alpha} \delta\left(t_{01}\right) \partial_{t_{2}}^{2 \beta} \delta\left(t_{02}\right) \partial_{t_{3}}^{2 \gamma} \delta\left(t_{03}\right) I_{3}^{D}[\hat{\alpha}, \hat{\beta}, \hat{\gamma}] .
\end{aligned}
$$

Here, $\Gamma_{\alpha}=\Gamma(\alpha)$ denotes the Gamma-function, we use the shorthand $\hat{\alpha}=D / 2-\alpha-1$ and we have introduced the following family of (Euclidean) integrals:

$$
I_{3}^{D}\left[a_{1}, a_{2}, a_{3}\right]:=\int \frac{\mathrm{d}^{D} \mathbf{x}_{0}}{\left(\mathbf{x}_{01}^{2}\right)^{a_{1}}\left(\mathbf{x}_{02}^{2}\right)^{a_{2}}\left(\mathbf{x}_{03}^{2}\right)^{a_{3}}} .
$$

These integrals represent the central nontrivial input for the above expansion (30) and we will now discuss how to compute them. Notably, in [78] the integrals $I_{3}^{D}\left[a_{1}, a_{2}, a_{3}\right]$ for generic propagator powers $a_{j}$ and spacetime dimension $D$ have been expressed in terms of Appell hypergeometric functions $F_{4}$, which converge for small values of the effective ratio variables $r_{12} / r_{13}$ and $r_{23} / r_{13}$. In the present situation we would like to avoid making assumptions on these ratios, which would imply a limited validity of the resulting effective potential. Moreover, note that here we are merely interested in the special case of half integer propagator powers $a_{j}$ in three dimensions, which satisfy the condition

$$
a_{1}+a_{2}+a_{3} \leq \frac{D}{2}
$$

In particular, this condition implies that the integrals of interest are divergent in strictly three dimensions and we thus consider their $\epsilon$-expansion around $D=3$ in dimensional regularization. Importantly, these integrals are accessible via a bootstrap approach, cf. $[68,72]$ : they feature a nonlocal Yangian level-one symmetry, i.e., they are annihilated by the differential operator 


$$
\hat{\mathrm{P}}^{\mu}:=\frac{i}{2} \sum_{k=1}^{3} \sum_{j=1}^{k-1}\left(\mathrm{P}_{j}^{\mu} \mathrm{D}_{k}+\mathrm{P}_{j \nu} \mathrm{L}_{k}^{\mu \nu}-(j \leftrightarrow k)\right)+\sum_{j=1}^{3} s_{j} \mathrm{P}_{j}^{\mu},
$$

where we have used the following representation of the momentum, Lorentz and dilatation generator of the conformal algebra:

$$
\begin{aligned}
\mathrm{P}_{j}^{\mu} & =-i \partial_{x_{j}}^{\mu}, \\
\mathrm{L}_{j}^{\mu \nu} & =i x_{j}^{\mu} \partial_{x_{j}}^{\nu}-i x_{j}^{\nu} \partial_{x_{j}}^{\mu}, \\
\mathrm{D}_{j} & =-i x_{j \mu} \partial_{x_{j}}^{\mu}-i .
\end{aligned}
$$

The so-called evaluation parameters $s_{j}$ entering the definition of the level-one generator $\hat{\mathrm{P}}^{\mu}$ in (33) take values [67]

$$
\left\{s_{j}\right\}=\frac{1}{2}\left\{a_{2}+a_{3}, a_{3}-a_{1},-a_{1}-a_{2}\right\} .
$$

Notably, in a dual momentum space, introduced via the transformation (19), i.e., $R_{j}=x_{j+1}-x_{j-1}$, the level-one generator $\hat{\mathrm{P}}$ translates into a representation of the special conformal generator [72]. Invariance under $\hat{\mathrm{P}}^{\mu}$ implies two independent partial differential equations (cf. [68] for the PDEs in terms of ratio variables)

$$
A_{1} I_{3}=0, \quad A_{2} I_{3}=0,
$$

with the second order differential operators

$$
\begin{aligned}
A_{1}= & +r_{12}\left(\bar{w}_{D}-2 a_{2}\right) \partial_{r_{13}}-2 r_{12} r_{23} \partial_{r_{13}} \partial_{r_{23}}-r_{12} r_{13} \partial_{r_{13}}^{2} \\
& +r_{13}\left(\bar{w}_{D}+2 a_{3}\right) \partial_{r_{12}}-2 r_{12}^{2} \partial_{r_{12}} \partial_{r_{13}}-r_{12} r_{13} \partial_{r_{12}}^{2} \\
A_{2}= & +r_{12}\left(\bar{w}_{D}+2 a_{1}\right) \partial_{r_{23}}-r_{12} r_{23} \partial_{r_{23}}^{2} \\
& -r_{23}\left(\bar{w}_{D}+2 a_{3}\right) \partial_{r_{12}}+r_{12} r_{23} \partial_{r_{12}}^{2} .
\end{aligned}
$$

Here, for the conformal weight of the integrals (31), we have introduced the abbreviation

$$
w_{D}=D-2\left(a_{1}+a_{2}+a_{3}\right)
$$

and $\bar{w}_{D}=w_{D}-1$. For $D=3-2 \epsilon$ we make the following ansatz for the $\epsilon$-expansion of the integral $I_{3}$, which is inspired by [79]:

$\mu^{-2 \epsilon} I_{3}^{3-2 \epsilon}=\frac{A}{2 \epsilon}+B+C \log \left(\frac{r_{12}+r_{13}+r_{23}}{\mu}\right)+\mathcal{O}(\epsilon)$.

Here $\mu$ denotes some mass scale and $A, B, C$ represent polynomials whose form is constrained by the scaling of the integral:

$$
X=\sum_{j=0}^{w_{3}} \sum_{k=0}^{w_{3}-j} f_{j k}^{(X)} r_{12}^{j} r_{13}^{k} r_{23}^{w_{3}-j-k}
$$

For $X \in\{A, B, C\}$ the constant coefficients of the polynomial are denoted by $f_{j k}^{(X)}$. We note that the polynomial $B$ can always be shifted by a term proportional to $C$ via a modification of the mass scale $\mu$. The below results are thus to be understood modulo such a shift. As the coefficients of $1 / \epsilon$ and $\log \mu$ are correlated in the $\epsilon$-expansion of (39), we must have $A=-C$ which we also find from the bootstrap arguments.

The solution of the homogeneous differential equations (36) will depend on some undetermined constants. In general, these can for instance be fixed by comparing a coincident point limit of the solution with the following well known expression for the two-point integral, cf. e.g., [80]:

$$
\int \frac{\mathrm{d}^{D} x_{0}}{x_{01}^{2 a_{1}} x_{02}^{2 a_{2}}}=\pi^{\frac{D}{2}} \frac{\Gamma_{a_{1}+a_{2}-\frac{D}{2}} \Gamma_{\frac{D}{2}-a_{1}} \Gamma_{\frac{D}{2}-a_{2}}}{\Gamma_{a_{1}} \Gamma_{a_{2}} \Gamma_{D-a_{1}-a_{2}}} r_{12}^{D-2 a_{1}-2 a_{2}} .
$$

However, for the lower propagator powers considered below, some of the arguments of the Gamma-functions will actually be zero. It is thus useful to note that the Laplacian acting on leg 1 of the integral generates a recursive structure on the above integrals, e.g.,

$$
\Delta_{1} I_{3}\left[a_{1}, a_{2}, a_{3}\right]=2 a_{1}\left(2 a_{1}+2-D\right) I_{3}\left[a_{1}+1, a_{2}, a_{3}\right],
$$

and similar for legs 2 and 3 . This equation can alternatively be used to relate the undetermined coefficients for integrals with negative propagator powers to the leading-order "seed" integral $I_{3}\left[\frac{1}{2}, \frac{1}{2}, \frac{1}{2}\right]$.

In the following we bootstrap the integrals contributing to the leading terms of the nonrelativistic expansion (30) using the level-one Yangian PDEs (37). We have compared the expansion of the below results for small ratios $r_{12} / r_{13}$ and $r_{23} / r_{13}$ to the expressions in terms of Appell hypergeometric functions given in [78] finding full agreement, see also [72] for our conventions. The following integrals serve as input for the three-body effective potential via (30) and (16).

\section{A. Order $c^{0}: I_{3}\left[\frac{1}{2}, \frac{1}{2}, \frac{1}{2}\right]$}

The leading order contribution to the expansion (30) is given by propagator powers $a_{j}=1 / 2$ for $j=1,2,3$. Using the ansatz (39) it is straightforward to solve the PDEs (37) in the $\epsilon$-expansion around $D=3$, which yields

$\mu^{-2 \epsilon} I_{3}\left[\frac{1}{2}, \frac{1}{2}, \frac{1}{2}\right]=\frac{b_{1}}{2 \epsilon}-b_{2} \log \left(\frac{r_{12}+r_{13}+r_{23}}{\mu}\right)+\mathcal{O}(\epsilon)$, 
for some undetermined constants $b_{1}, b_{2}$. The parameters $b_{1}, b_{2}$ are fixed by comparison with the two-point integral (41) to $b_{1}=b_{2}=4 \pi$. Note that we do not display an additional constant that can be shifted by modification of the mass scale $\mu$. The above logarithmic result for this integral is already contained in [79].

\section{B. Order $c^{-2}: I_{3}\left[\frac{1}{2}, \frac{1}{2},-\frac{1}{2}\right]$}

In complete analogy to the above, we find at next-toleading order the following $\epsilon$-expansion of the single contributing integral:

$$
\begin{aligned}
& \mu^{-2 \epsilon} I_{3}\left[\frac{1}{2}, \frac{1}{2},-\frac{1}{2}\right] \\
& =-\frac{2 \pi}{3}\left[\frac{r_{12}^{2}-r_{13}^{2}-r_{23}^{2}}{2 \epsilon}-\left(r_{12}-r_{13}\right)\left(r_{12}-r_{23}\right)\right. \\
& \left.\quad-\left(r_{12}^{2}-r_{13}^{2}-r_{23}^{2}\right) \log \left(\frac{r_{12}+r_{13}+r_{23}}{\mu}\right)\right]+\mathcal{O}(\epsilon) .
\end{aligned}
$$

Below we will employ this result to obtain new contributions to the three-body effective potential at 3PN, which scale as $v^{4} G^{2} m^{3} / c^{8} r^{2}$.

$$
\text { C. Order } c^{-4}: I_{3}\left[-\frac{1}{2},-\frac{1}{2}, \frac{1}{2}\right] \text { and } I_{3}\left[\frac{1}{2}, \frac{1}{2},-\frac{3}{2}\right]
$$

To demonstrate that the above bootstrap approach easily generalizes to higher orders, let us also consider the next order. Note, however, that due to its length we will not evaluate the resulting contribution to the effective potential in this paper, see Sec. VII for the previous order. At order $c^{-4}$ of the nonrelativistic expansion (30) two integrals contribute. With the ansatz (39) we can again solve the above partial differential equations to find solutions of the form

$\mu^{-2 \epsilon} I_{3}^{3-2 \epsilon}=b\left[\frac{A}{2 \epsilon}-B-A \log \left(\frac{r_{12}+r_{13}+r_{23}}{\mu}\right)\right]+\mathcal{O}(\epsilon)$.

Note again that the polynomial $B$ is only defined modulo a shift by $A$ due to the arbitrariness of the mass scale $\mu$. Here we have

$$
\begin{aligned}
A\left[-\frac{1}{2},-\frac{1}{2}, \frac{1}{2}\right]= & -\frac{1}{3} r_{12}^{4}+\left(r_{13}^{2}-r_{23}^{2}\right)^{2}-\frac{2}{3} r_{12}^{2}\left(r_{13}^{2}+r_{23}^{2}\right), \\
B\left[-\frac{1}{2},-\frac{1}{2}, \frac{1}{2}\right]= & \frac{r_{12}^{4}}{9}-r_{12}\left(r_{13}-r_{23}\right)^{2}\left(r_{13}+r_{23}\right) \\
& +\frac{1}{3} r_{12}^{3}\left(r_{13}+r_{23}\right)-r_{13} r_{23}\left(r_{13}-r_{23}\right)^{2} \\
& +\frac{1}{9} r_{12}^{2}\left(5 r_{13}^{2}-3 r_{13} r_{23}+5 r_{23}^{2}\right),
\end{aligned}
$$

as well as

$$
\begin{aligned}
A\left[\frac{1}{2}, \frac{1}{2},-\frac{3}{2}\right]= & -r_{12}^{4}-r_{13}^{4}-r_{23}^{4} \\
& +2 r_{12}^{2}\left(r_{13}^{2}+r_{23}^{2}\right)-\frac{2}{3} r_{13}^{2} r_{23}^{2}, \\
B\left[\frac{1}{2}, \frac{1}{2},-\frac{3}{2}\right]= & -\frac{4 r_{12}^{4}}{3}+r_{13} r_{23}^{3}-\frac{4}{9} r_{13}^{2} r_{23}^{2}+r_{13}^{3} r_{23} \\
& +r_{12}^{3}\left(r_{13}+r_{23}\right)+r_{12}^{2}\left(2 r_{13}^{2}-r_{23} r_{13}+2 r_{23}^{2}\right) \\
& -\frac{1}{3} r_{12}\left(5 r_{13}^{3}+3 r_{23} r_{13}^{2}+3 r_{23}^{2} r_{13}+5 r_{23}^{3}\right) .
\end{aligned}
$$

The overall constants $b$ in (45) are fixed by relating them to the coefficients for the seed integral (43) via the recursion (42):

$$
b\left[-\frac{1}{2},-\frac{1}{2}, \frac{1}{2}\right]=-\frac{\pi}{10}, \quad b\left[\frac{1}{2}, \frac{1}{2},-\frac{3}{2}\right]=-\frac{3 \pi}{10} .
$$

This provides all the necessary information to generate the 4PN order $G^{2}$ contributions to the effective potential.

\section{THE 2PN EXPANSION}

We now proceed to compute the $2 \mathrm{PN}$ expansion of the effective action as introduced in Sec. IV. This also serves as a test for the integral (43). For the third line of (16), we decompose the sum as

$$
\sum_{i, j, k}^{\prime} \rightarrow \sum_{i} \sum_{j \neq i} \sum_{k \neq i, j}+\left(\left.\sum_{i} \sum_{j}\right|_{k=i}+(\text { cyclic })\right) .
$$

Here we refer to the first term on the right-hand side as the three-body interaction and to the remaining terms as the two-body interactions. When identifying two of the three indices, we encounter a divergence $1 /\left.r_{i j}\right|_{j=i}$ and an indefinite unit vector $\left.\mathbf{n}_{i j}\right|_{j=i}$. In light of the vanishing of propagators with both ends on the same worldline, we propose to regularize the divergences as $1 /\left.r_{i j}\right|_{j=i} \rightarrow 0$. Terms of odd order in $\left.\mathbf{n}_{i j}\right|_{j=i}$ also vanish due to the antisymmetry in the indices. For the quadratic terms in $\left.\mathbf{n}_{i j}\right|_{j=i}$ of the 2 PN result we adopt the following limiting prescription:

$$
\left.\mathbf{n}_{i j} \cdot \mathbf{v}_{\alpha} \mathbf{n}_{i j} \cdot \mathbf{v}_{\beta}\right|_{j=i} \rightarrow \mathbf{v}_{\alpha} \cdot \mathbf{v}_{\beta}
$$

That is, whenever the identification of two points yields an expression as given on the left-hand side, we replace it by the right-hand side. This prescription is natural from the perspective of dimensional analysis and symmetry 
considerations, and it reproduces the correct results as given in the literature. With regard to the 3PN result to be discussed in Sec. VII we already give the rule

$$
\begin{aligned}
\mathbf{n}_{i j} & \left.\cdot \mathbf{v}_{\alpha} \mathbf{n}_{i j} \cdot \mathbf{v}_{\beta} \mathbf{n}_{i j} \cdot \mathbf{v}_{\rho} \mathbf{n}_{i j} \cdot \mathbf{v}_{\sigma}\right|_{j=i} \\
& \rightarrow \mathbf{v}_{\alpha} \cdot \mathbf{v}_{\beta} \mathbf{v}_{\rho} \cdot \mathbf{v}_{\sigma}+\mathbf{v}_{\alpha} \cdot \mathbf{v}_{\rho} \mathbf{v}_{\sigma} \cdot \mathbf{v}_{\beta}+\mathbf{v}_{\alpha} \cdot \mathbf{v}_{\sigma} \mathbf{v}_{\beta} \cdot \mathbf{v}_{\rho} .
\end{aligned}
$$

We note that the $1 / \epsilon$-term in (43) naturally drops out in the final expression for the action due to the derivatives that have to be applied. Moreover, we expect this property to hold to all orders in the PN expansion. This is explicitly shown to be true in the 3PN calculation of Sec. VII. The 2PN effective action reads

$$
\begin{aligned}
S^{2 \mathrm{PN}}= & \sum_{i} \int \frac{\mathrm{d} t}{c^{6}}\left\{\frac{m_{i} \mathbf{v}_{i}^{6}}{16}+\sum_{j \neq i} \frac{G m_{i} m_{j}}{16 r_{i j}}\left[3\left(\mathbf{n}_{i j} \cdot \mathbf{v}_{i}\right)^{2}\left(\mathbf{n}_{i j} \cdot \mathbf{v}_{j}\right)^{2}-6 \mathbf{n}_{i j} \cdot \mathbf{v}_{i} \mathbf{n}_{i j} \cdot \mathbf{v}_{j} \mathbf{v}_{i j}^{2}-2\left(\mathbf{n}_{i j} \cdot \mathbf{v}_{j}\right)^{2} \mathbf{v}_{i}^{2}\right.\right. \\
& \left.+3 \mathbf{v}_{i}^{2} \mathbf{v}_{j}^{2}+2\left(\mathbf{v}_{i} \cdot \mathbf{v}_{j}\right)^{2}-20 \mathbf{v}_{i}^{2} \mathbf{v}_{i} \cdot \mathbf{v}_{j}+14 \mathbf{v}_{i}^{4}\right]+\sum_{j \neq i} \frac{G^{2} m_{i} m_{j}^{2}}{2 r_{i j}^{2}}\left[33\left(\mathbf{n}_{i j} \cdot \mathbf{v}_{i j}\right)^{2}-17 \mathbf{v}_{i j}^{2}\right] \\
& +\sum_{j \neq i} \sum_{k \neq i} \frac{G^{2} m_{i} m_{j} m_{k}}{8}\left[\frac{1}{r_{i j} r_{i k}}\left(4\left(\mathbf{n}_{i j} \cdot \mathbf{v}_{j}\right)^{2}+18 \mathbf{v}_{i}^{2}-16 \mathbf{v}_{j}^{2}-32 \mathbf{v}_{i} \cdot \mathbf{v}_{j}+32 \mathbf{v}_{j} \cdot \mathbf{v}_{k}\right)\right. \\
& \left.+\frac{1}{r_{i j}^{2}}\left(14 \mathbf{n}_{i k} \cdot \mathbf{v}_{k} \mathbf{n}_{i j} \cdot \mathbf{v}_{k}-12 \mathbf{n}_{i j} \cdot \mathbf{v}_{i} \mathbf{n}_{i k} \cdot \mathbf{v}_{k}+\mathbf{n}_{i j} \cdot \mathbf{n}_{i k}\left(\mathbf{n}_{i k} \cdot \mathbf{v}_{k}\right)^{2}-\mathbf{n}_{i j} \cdot \mathbf{n}_{i k} \mathbf{v}_{k}^{2}\right)\right] \\
& +\sum_{j \neq i} \sum_{k \neq i, j} G^{2} m_{i} m_{j} m_{k}\left[\frac{2\left(\mathbf{n}_{i j}-\mathbf{n}_{j k}\right) \cdot \mathbf{v}_{i j}}{\left(r_{i j}+r_{i k}+r_{j k}\right)^{2}}\left(4\left(\mathbf{n}_{i j}+\mathbf{n}_{i k}\right) \cdot \mathbf{v}_{i j}+\left(\mathbf{n}_{i k}+\mathbf{n}_{j k}\right) \cdot \mathbf{v}_{i k}\right)\right. \\
& +\frac{\left.\left.9\left(\mathbf{n}_{i j} \cdot \mathbf{v}_{i j}\right)^{2}-9 \mathbf{v}_{i j}^{2}+2\left(\mathbf{n}_{i j} \cdot \mathbf{v}_{i k}\right)^{2}-2 \mathbf{v}_{i k}^{2}\right]\right\}+G^{3} \times[\text { static term }],}{r_{i j}\left(r_{i j}+r_{i k}+r_{j k}\right)}
\end{aligned}
$$

where we define $\mathbf{v}_{i j}:=\mathbf{v}_{i}-\mathbf{v}_{j}$. Here we have performed a field redefinition to push terms that involve accelerations to higher orders in $G$. We have checked that our result agrees with the literature $[47,48]$ up to a total derivative. Note that we do not have access to the static (velocity independent) term at $\mathcal{O}\left(G^{3}\right)$ in our approach as it stems from a 3PM computation.

\section{NEW CONTRIBUTIONS AT 3PN}

In this section we explicitly evaluate the contributions to the 3PN three-body effective potential. Limiting the number of point masses to two gives the two-body 3PN effective action, which we checked to agree with [27] up to a total derivative. Next to the novel three-point $G^{2} v^{4}$ terms, the below expression contains terms that scale as $G v^{6}$, as well as two-point terms of order $G^{2} v^{4}$ which have been known before. The full 3PN action can be written in the form

$$
\begin{aligned}
S^{3 \mathrm{PN}}= & \sum_{i} \int \frac{\mathrm{d} t}{c^{8}}\left\{\frac{5}{128} m_{i} \mathbf{v}_{i}^{8}+L_{(A)}^{3 \mathrm{PN}}+L_{(B)}^{3 \mathrm{PN}}\right. \\
& \left.+L_{(C)}^{3 \mathrm{PN}}+L_{(D)}^{3 \mathrm{PN}}\right\}+\mathcal{O}\left(G^{3}\right) .
\end{aligned}
$$

Note that the terms at order $G^{3}$ are not given here and require two yet unknown four-point integrals at one and two loops. Moreover, there are additional $G^{4}$ contributions at 3PN. In (53) we have ordered the various terms, which are explicitly given in the following, by their power of $G$ and the structure of summations. Terms from perturbative solutions of the equations of motion for the einbein, cf. (22), contribute at various places. Explicit expressions for the terms in (53) are also provided in an ancillary file to this paper [81]. The term $L_{(A)}^{3 \mathrm{PN}}$ originates from the $1 \mathrm{PM}$ action and reads

$$
\begin{aligned}
L_{(A)}^{3 \mathrm{PN}}= & \sum_{j \neq i} \frac{G m_{i} m_{j}}{32 r_{i j}}\left[-5\left(\mathbf{n}_{i j} \cdot \mathbf{v}_{i}\right)^{3}\left(\mathbf{n}_{i j} \cdot \mathbf{v}_{j}\right)^{3}+3 \mathbf{n}_{i j} \cdot \mathbf{v}_{i}\left(\mathbf{n}_{i j} \cdot \mathbf{v}_{j}\right)^{2}\left(2 \mathbf{v}_{i}^{2} \mathbf{n}_{i j} \cdot \mathbf{v}_{j}+6 \mathbf{v}_{i}^{2} \mathbf{n}_{i j} \cdot \mathbf{v}_{i}-5 \mathbf{v}_{i} \cdot \mathbf{v}_{j} \mathbf{n}_{i j} \cdot \mathbf{v}_{i}\right)\right. \\
& +\mathbf{n}_{i j} \cdot \mathbf{v}_{i} \mathbf{n}_{i j} \cdot \mathbf{v}_{j}\left(10\left(\mathbf{v}_{i} \cdot \mathbf{v}_{j}\right)^{2}+8 \mathbf{v}_{i}^{2} \mathbf{v}_{i} \cdot \mathbf{v}_{j}-5 \mathbf{v}_{i}^{2} \mathbf{v}_{j}^{2}-14 \mathbf{v}_{i}^{4}\right)+2\left(\mathbf{n}_{i j} \cdot \mathbf{v}_{j}\right)^{2} \mathbf{v}_{i}^{2}\left(5 \mathbf{v}_{i} \cdot \mathbf{v}_{j}-3 \mathbf{v}_{i}^{2}\right) \\
& \left.-6 \mathbf{v}_{i}^{2} \mathbf{v}_{j}^{2}\left(\mathbf{n}_{i j} \cdot \mathbf{v}_{i}\right)^{2}+16 \mathbf{v}_{i}^{4} \mathbf{v}_{j}^{2}+2\left(\mathbf{v}_{i} \cdot \mathbf{v}_{j}\right)^{3}+12 \mathbf{v}_{i}^{2}\left(\mathbf{v}_{i} \cdot \mathbf{v}_{j}\right)^{2}-19 \mathbf{v}_{i}^{2} \mathbf{v}_{j}^{2} \mathbf{v}_{i} \cdot \mathbf{v}_{j}-34 \mathbf{v}_{i}^{4} \mathbf{v}_{i} \cdot \mathbf{v}_{j}+22 \mathbf{v}_{i}^{6}\right] .
\end{aligned}
$$

Here we have added a total derivative as given in (C1) in Appendix C. Again, accelerations have been pushed to the next order in $G$ by means of field redefinitions. The next term $L_{(B)}^{3 \mathrm{PN}}$ stems from the two-body interactions of the third line of (16) and reads 


$$
\begin{aligned}
L_{(B)}^{3 \mathrm{PN}}= & \sum_{j \neq i} \frac{G^{2} m_{i} m_{j}^{2}}{4 r_{i j}^{2}}\left[\left(-200 \mathbf{v}_{i} \cdot \mathbf{v}_{j}+167 \mathbf{v}_{i}^{2}+66 \mathbf{v}_{j}^{2}\right)\left(\mathbf{n}_{i j} \cdot \mathbf{v}_{i}\right)^{2}-2\left(99 \mathbf{v}_{i}^{2}+64 \mathbf{v}_{j}^{2}-130 \mathbf{v}_{i} \cdot \mathbf{v}_{j}\right) \mathbf{n}_{i j} \cdot \mathbf{v}_{j} \mathbf{n}_{i j} \cdot \mathbf{v}_{i}\right. \\
& -44\left(\mathbf{n}_{i j} \cdot \mathbf{v}_{i}-\mathbf{n}_{i j} \cdot \mathbf{v}_{j}\right)^{2}\left(2\left(\mathbf{n}_{i j} \cdot \mathbf{v}_{i}\right)^{2}+\left(\mathbf{n}_{i j} \cdot \mathbf{v}_{j}\right)^{2}\right)+\left(65 \mathbf{v}_{i}^{2}+96 \mathbf{v}_{j}^{2}-128 \mathbf{v}_{i} \cdot \mathbf{v}_{j}\right)\left(\mathbf{n}_{i j} \cdot \mathbf{v}_{j}\right)^{2} \\
& \left.-98\left(\mathbf{v}_{i} \cdot \mathbf{v}_{j}\right)^{2}+96 \mathbf{v}_{j}^{2} \mathbf{v}_{i} \cdot \mathbf{v}_{j}+\mathbf{v}_{i}^{2}\left(134 \mathbf{v}_{i} \cdot \mathbf{v}_{j}-49 \mathbf{v}_{j}^{2}\right)-51 \mathbf{v}_{i}^{4}-32 \mathbf{v}_{j}^{4}\right]
\end{aligned}
$$

Moreover, the term $L_{(C)}^{3 \mathrm{PN}}$ to (53) receives contributions from the second line of (16) as well as from field redefinitions and total derivatives that we use to remove terms that involve accelerations:

$$
\begin{aligned}
L_{(C)}^{3 \mathrm{PN}}= & \sum_{j \neq i} \sum_{k \neq i} \frac{G^{2} m_{i} m_{j} m_{k}}{16}\left\{\frac { 1 } { r _ { i j } r _ { i k } } \left[2\left(\mathbf{n}_{i j} \cdot \mathbf{v}_{j}\right)^{2}\left(16 \mathbf{v}_{i} \cdot \mathbf{v}_{j}-18 \mathbf{v}_{i}^{2}-32 \mathbf{v}_{j} \cdot \mathbf{v}_{k}+12 \mathbf{v}_{j}^{2}-\left(\mathbf{n}_{i k} \cdot \mathbf{v}_{k}\right)^{2}\right)\right.\right. \\
& +64 \mathbf{v}_{i} \cdot \mathbf{v}_{j}\left(2\left(\mathbf{n}_{i k} \cdot \mathbf{v}_{k}\right)^{2}+\mathbf{v}_{i} \cdot \mathbf{v}_{k}-2 \mathbf{v}_{j} \cdot \mathbf{v}_{k}-\mathbf{v}_{j}^{2}\right)+16 \mathbf{v}_{j}^{2}\left(8 \mathbf{v}_{j} \cdot \mathbf{v}_{k}-2 \mathbf{v}_{j}^{2}-2 \mathbf{v}_{k}^{2}-\left(\mathbf{n}_{i k} \cdot \mathbf{v}_{k}\right)^{2}\right) \\
& \left.+16 \mathbf{v}_{i}^{2}\left(3 \mathbf{v}_{j}^{2}+2 \mathbf{v}_{j} \cdot \mathbf{v}_{k}-10 \mathbf{v}_{i} \cdot \mathbf{v}_{j}\right)-6\left(\mathbf{n}_{i j} \cdot \mathbf{v}_{j}\right)^{4}+96\left(\mathbf{v}_{i} \cdot \mathbf{v}_{j}\right)^{2}+49 \mathbf{v}_{i}^{4}\right]+\frac{1}{3 r_{i j}^{2}}\left[20\left(\mathbf{n}_{i k} \cdot \mathbf{v}_{k}\right)^{3}\left(\mathbf{n}_{i j} \cdot \mathbf{v}_{i}-\mathbf{n}_{i j} \cdot \mathbf{v}_{k}\right)\right. \\
& -3 \mathbf{n}_{i j} \cdot \mathbf{n}_{i k}\left(\left(\mathbf{n}_{i k} \cdot \mathbf{v}_{k}\right)^{2}-\mathbf{v}_{k}^{2}\right)\left(3\left(\mathbf{n}_{i j} \cdot \mathbf{v}_{j}\right)^{2}+8 \mathbf{v}_{i} \cdot \mathbf{v}_{j}+\left(\mathbf{n}_{i k} \cdot \mathbf{v}_{k}\right)^{2}+2 \mathbf{n}_{i k} \cdot \mathbf{v}_{i} \mathbf{n}_{i k} \cdot \mathbf{v}_{k}+6 \mathbf{v}_{i} \cdot \mathbf{v}_{k}-5 \mathbf{v}_{i}^{2}-4 \mathbf{v}_{j}^{2}-4 \mathbf{v}_{k}^{2}\right) \\
& +6\left(\left(\mathbf{n}_{i k} \cdot \mathbf{v}_{k}\right)^{2}-\mathbf{v}_{k}^{2}\right)\left(3 \mathbf{n}_{i j} \cdot \mathbf{v}_{j} \mathbf{n}_{i k} \cdot \mathbf{v}_{i}-3 \mathbf{n}_{i j} \cdot \mathbf{v}_{j} \mathbf{n}_{i k} \cdot \mathbf{v}_{j}-3 \mathbf{n}_{i k} \cdot \mathbf{v}_{i} \mathbf{n}_{i j} \cdot \mathbf{v}_{k}+4 \mathbf{n}_{i j} \cdot \mathbf{v}_{i} \mathbf{n}_{i k} \cdot \mathbf{v}_{j}-\mathbf{n}_{i j} \cdot \mathbf{v}_{i} \mathbf{n}_{i k} \cdot \mathbf{v}_{i}\right) \\
& +6 \mathbf{n}_{i k} \cdot \mathbf{v}_{k}\left[\mathbf{n}_{i j} \cdot \mathbf{v}_{k}\left(19 \mathbf{v}_{i}^{2}+28 \mathbf{v}_{j}^{2}-56 \mathbf{v}_{i} \cdot \mathbf{v}_{j}-2 \mathbf{v}_{i} \cdot \mathbf{v}_{k}-21\left(\mathbf{n}_{i j} \cdot \mathbf{v}_{j}\right)^{2}\right)+6 \mathbf{n}_{i j} \cdot \mathbf{v}_{j}\left(6 \mathbf{v}_{i} \cdot \mathbf{v}_{j}+7 \mathbf{v}_{i} \cdot \mathbf{v}_{k}-6 \mathbf{v}_{i}^{2}-7 \mathbf{v}_{j} \cdot \mathbf{v}_{k}\right)\right. \\
& \left.\left.\left.+2 \mathbf{n}_{i j} \cdot \mathbf{v}_{i}\left(11 \mathbf{v}_{i}^{2}-12 \mathbf{v}_{j}^{2}-23 \mathbf{v}_{i} \cdot \mathbf{v}_{k}+28 \mathbf{v}_{j} \cdot \mathbf{v}_{k}+9\left(\mathbf{n}_{i j} \cdot \mathbf{v}_{j}\right)^{2}\right)\right]+18 \mathbf{v}_{k}^{2} \mathbf{n}_{i k} \cdot \mathbf{v}_{k}\left(5 \mathbf{n}_{i j} \cdot \mathbf{v}_{k}-4 \mathbf{n}_{i j} \cdot \mathbf{v}_{i}\right)\right]\right\} .
\end{aligned}
$$

The term $L_{(D)}^{3 \mathrm{PN}}$ contributing to the above action originates from the three-body parts of the third line of (16) and can be expressed in terms of derivatives that act on the integrals $I_{3}\left[\frac{1}{2}, \frac{1}{2}, \frac{1}{2}\right]$ and $I_{3}\left[\frac{1}{2}, \frac{1}{2},-\frac{1}{2}\right]$ as given in (43) and (44) of Sec. V:

$$
\begin{aligned}
L_{(D)}^{3 \mathrm{PN}}= & \sum_{j \neq i} \sum_{k \neq i, j} \frac{G^{2} m_{i} m_{j} m_{k}}{4 \pi}\left\{\left[\left(6 \mathbf{v}_{i}^{2}+\mathbf{v}_{k}^{2}-8 \mathbf{v}_{i} \cdot \mathbf{v}_{j}\right)\left(\mathbf{v}_{k i} \cdot \partial_{x_{i}}\right)\left(\mathbf{v}_{k j} \cdot \partial_{x_{j}}\right)+\left(8 \mathbf{v}_{i k}^{2}-4 \mathbf{v}_{k}^{2}\right)\left(\mathbf{v}_{j i} \cdot \partial_{x_{i}}\right)\left(\mathbf{v}_{i j} \cdot \partial_{x_{j}}\right)\right] I_{3}\left[\frac{1}{2}, \frac{1}{2}, \frac{1}{2}\right]\right. \\
& +\left(\mathbf{v}_{k} \cdot \partial_{x_{k}}\right)^{2}\left[\left(\mathbf{v}_{k i} \cdot \partial_{x_{i}}\right)\left(\mathbf{v}_{k j} \cdot \partial_{x_{j}}\right)+2\left(\mathbf{v}_{i k} \cdot \partial_{x_{k}}\right)\left(\mathbf{v}_{i j} \cdot \partial_{x_{j}}\right)+4\left(\mathbf{v}_{j i} \cdot \partial_{x_{i}}\right)\left(\mathbf{v}_{i j} \cdot \partial_{x_{j}}\right)\right. \\
& \left.\left.+8\left(\mathbf{v}_{j k} \cdot \partial_{x_{k}}\right)\left(\mathbf{v}_{k j} \cdot \partial_{x_{j}}\right)\right] I_{3}\left[\frac{1}{2}, \frac{1}{2},-\frac{1}{2}\right]\right\}
\end{aligned}
$$

Here the integrals $I_{3}$ depend on the three external points $i, j, k$ as opposed to Sec. V, where the labels $1,2,3$ were used. For convenience we display again the expressions (43) and (44):

$$
\mu^{-2 \epsilon} I_{3}\left[\frac{1}{2}, \frac{1}{2}, \frac{1}{2}\right]=\frac{2 \pi}{\epsilon}-4 \pi \log \left(\frac{r_{i j}+r_{i k}+r_{j k}}{\mu}\right)+\mathcal{O}(\epsilon)
$$

$\mu^{-2 \epsilon} I_{3}\left[\frac{1}{2}, \frac{1}{2},-\frac{1}{2}\right]=-\frac{2 \pi}{3}\left[\frac{r_{i j}^{2}-r_{i k}^{2}-r_{j k}^{2}}{2 \epsilon}-\left(r_{i j}-r_{i k}\right)\left(r_{i j}-r_{j k}\right)-\left(r_{i j}^{2}-r_{i k}^{2}-r_{j k}^{2}\right) \log \left(\frac{r_{i j}+r_{i k}+r_{j k}}{\mu}\right)\right]+\mathcal{O}(\epsilon)$

Note again that by identifying two indices in the above $L_{(D)}^{3 \mathrm{PN}}$ and using the prescriptions given in (50) and (51) we obtain the corresponding two-body contributions (55). The $1 / \epsilon$-poles and the mass scale $\mu$ in the expressions for the integrals $I_{3}$ drop out after taking the derivatives in (57). This property persists at least to the 4PN order.
The structure of the result for $L_{(D)}^{3 \mathrm{PN}}$ after evaluating these derivatives is displayed in Appendix C.

Note that the contribution from the second line of (16), which is already contained in the above expression (56) for $L_{(C)}^{3 \mathrm{PN}}$, can also explicitly be written in the form before taking derivatives 


$$
\begin{aligned}
L_{(\text {second })}^{3 \text { PN }}= & \sum_{j \neq i} \sum_{k \neq i} G^{2} m_{i} m_{j} m_{k}\left\{\frac { 1 } { 8 r _ { i j } r _ { i k } } \left[\mathbf{v}_{i}^{2}\left(\mathbf{v}_{i}^{2}-20 \mathbf{v}_{j}^{2}+16 \mathbf{v}_{j} \cdot \mathbf{v}_{k}\right)+2 \mathbf{v}_{j}^{2}\left(32 \mathbf{v}_{i} \cdot \mathbf{v}_{k}+16 \mathbf{v}_{j} \cdot \mathbf{v}_{k}-7 \mathbf{v}_{j}^{2}-9 \mathbf{v}_{k}^{2}\right)\right.\right. \\
& \left.+32 \mathbf{v}_{i} \cdot \mathbf{v}_{j}\left(\mathbf{v}_{i} \cdot \mathbf{v}_{j}-\mathbf{v}_{i} \cdot \mathbf{v}_{k}-2 \mathbf{v}_{j} \cdot \mathbf{v}_{k}\right)\right]+\left(\mathbf{v}_{i}^{2}-3 \mathbf{v}_{j}^{2}-3 \mathbf{v}_{k}^{2}+8 \mathbf{v}_{j} \cdot \mathbf{v}_{k}\right)\left(\mathbf{v}_{k} \cdot \partial_{x_{k}}\right)^{2} \frac{r_{i k}}{2 r_{i j}} \\
& \left.-\left[\left(\mathbf{v}_{k} \cdot \partial_{x_{k}}\right)^{2}\left(\mathbf{v}_{j} \cdot \partial_{x_{j}}\right)^{2} \frac{r_{i j} r_{i k}}{4}+\left(\mathbf{v}_{k} \cdot \partial_{x_{k}}\right)^{4} \frac{r_{i k}^{3}}{12 r_{i j}}\right]\right\} .
\end{aligned}
$$

This completes the details describing the final result (53) for the $G^{2}$ contributions to the $3 \mathrm{PN}$ effective action. We note again that in principle we could proceed with the same method to compute higher order contributions to the effective potential of order $G^{2} v^{2 n}$. The two new integrals that contribute to the next order of the expansion were already given in Sec. V. However, due to the length of the above terms at $G^{2} v^{4}$, see also the expanded result in Appendix C, we refrain here from explicitly evaluating the contributions at the next order $G^{2} v^{6}$.

\section{CONCLUSIONS AND OUTLOOK}

In the present paper we have extended the known results for the three-body effective potential in general relativity as follows:

(i) At order 2PM the potential is given by (17) expressed via a differential operator that acts on the three-point integral $I_{3 \delta}$ evaluated in Appendix A.

(ii) At $3 \mathrm{PN}$, new $G^{2} v^{4}$-contributions were obtained in Sec. VII and are explicitly provided in an ancillary file to this paper [81].

(iii) The key integrals contributing to the effective potential at $G^{2} v^{2 n}$ can be obtained by the bootstrap approach described in Sec. V. Due to their length, we here refrain from evaluating the resulting expressions for the effective potential.

There are a number of interesting directions that should be further explored. First, it would be important to establish the connection between the above PN results and the direct nonrelativistic expansion of the (integrated) expression (17) at 2PM. Approaching this problem one faces the lengthy distributional expressions given in Appendix B, whose PN expansion appears to require some regularization of diverging contributions. For this reason we have performed the PN expansion at the level of the 2PM integrand, and then evaluated the integrals. Still, rederiving the PN expansion from the final 2PM expression would represent an important cross check of the result. This should also be useful to understand the interplay of the different kinematical regions for the integral discussed in Appendix A.

With regard to the higher order PN contributions which scale as $G^{2} v^{2 n}$, it would be interesting to bootstrap a closed formula for the $\epsilon$-expansion of the family of integrals (31) around three dimensions. Exploiting the Yangian level-one symmetry discussed above, this should be feasible along the lines of [68]. Here it would be great to prove that the observed mechanism which makes the divergent contributions drop out in the final expression for the potential persists to all orders. Similarly, it should be explored how far these bootstrap methods reach in obtaining integrals at higher orders of the PM or PN expansion.

The Yangian level-one symmetry that we employed can also be understood as a special conformal symmetry in a dual momentum space. Here the dualized momenta relate to the above position space variables via (19) (not via Fourier transform). It would be interesting to investigate the employed symmetry in Fourier space and to see if there is a relation to the curious conformal symmetry of graviton amplitudes observed in [82].

Finally, one should see if one can feed the above contributions to the effective potential into numerical simulations updating the studies of $[8,19,20,22]$. Eventually it would be fascinating if the effect of the three-body interactions obtained here could be observed in the future.

\section{ACKNOWLEDGMENTS}

We would like to thank J. Bicak, M. Levi and G. Schäfer for helpful communications and R. Gonzo for discussions. This project has received funding from the European Union's Horizon 2020 research and innovation program under the Marie Sklodowska-Curie grant agreement No. 764850. The work of J.P. and T. W. is funded by the Deutsche Forschungsgemeinschaft (DFG, German Research Foundation)-Projektnummer PL457/3-1. The work of F. L. is funded by the Deutsche Forschungsgemeinschaft (DFG, German Research Foundation)_-Projektnummer 363895012.

\section{APPENDIX A: THE $3 \delta$ INTEGRAL}

Here we present a detailed calculation of the $3 \delta$-integral given by

$$
I_{3 \delta}=\int \mathrm{d}^{4} x_{0} \delta\left(x_{01}^{2}\right) \delta\left(x_{02}^{2}\right) \delta\left(x_{03}^{2}\right) .
$$

Recall $\sigma^{2}=\left(R_{2} \cdot R_{3}\right)^{2}-R_{2}^{2} R_{3}^{2}$ as defined in (20). Importantly, the quantity $-\sigma^{2}$ may be seen as the square of the area of the parallelogram spanned by $x_{1}, x_{2}, x_{3}$ and 
thus characterizes the space $\mathcal{M}$ spanned by these three points:

$$
\begin{aligned}
& \sigma^{2}>0, \quad \mathcal{M} \text { is } 2 \mathrm{D} \text { Minkowskian, } \\
& \sigma^{2}=0, \quad \mathcal{M} \text { is a } 1 \mathrm{D} \text { straight line, } \\
& \sigma^{2}<0, \quad \mathcal{M} \text { is } 2 \mathrm{D} \text { Euclidean. }
\end{aligned}
$$

We now explicitly evaluate the above integral for these three cases, generalizing the computation of Westpfahl [59] for the three-point integral with retarded propagators.

\section{The case $\sigma^{2}>0$}

We choose four basis vectors $R_{2}^{\mu}, R_{3}^{\mu}, \xi_{1}^{\mu}, \xi_{2}^{\mu}$ such that we can express the integration vector as

$x_{01}^{\mu}=\tau R_{2}^{\mu}+\bar{\tau} R_{3}^{\mu}+r\left(\cos \varphi \xi_{1}^{\mu}+\sin \varphi \xi_{2}^{\mu}\right), \quad r \geq 0$.

Here $\left\{\xi_{1}, \xi_{2}\right\}$ denotes the (orthogonal) unit basis of the perpendicular complement of $\mathcal{M}$ :

$$
\xi_{i} \cdot R_{j}=0, \quad \xi_{i} \cdot \xi_{j}=-\delta_{i j}, \quad \text { for } i, j=1,2 .
$$

In these coordinates the integration measure reads

$$
\mathrm{d}^{4} x_{0}=\frac{1}{2} \sigma \mathrm{d} \tau \mathrm{d} \bar{\tau} \mathrm{d} r^{2} \mathrm{~d} \varphi
$$

and the integral simplifies to

$$
\begin{aligned}
& \int \frac{\mathrm{d}^{4} x_{0}}{4 \sigma^{2}} \delta\left(r^{2}+\frac{R_{1}^{2} R_{2}^{2} R_{3}^{2}}{4 \sigma^{2}}\right) \delta\left(\tau-\frac{R_{3}^{2} R_{1} \cdot R_{2}}{2 \sigma^{2}}\right) \\
& \quad \times \delta\left(\bar{\tau}+\frac{R_{2}^{2} R_{1} \cdot R_{3}}{2 \sigma^{2}}\right) .
\end{aligned}
$$

This straightforwardly yields

$$
I_{3 \delta}\left(\sigma^{2}>0\right)=\frac{\pi}{4 \sigma} \Theta\left(-R_{1}^{2} R_{2}^{2} R_{3}^{2}\right) .
$$

Here $\Theta$ denotes the Heaviside step function defined as

$$
\Theta(x)= \begin{cases}1, & \text { for } x>0 \\ \frac{1}{2}, & \text { for } x=0 \\ 0, & \text { for } x<0\end{cases}
$$

Hence, we conclude that in the region $\sigma^{2}>0$ the piecewise constant in (20) is given by

$$
C\left(\sigma^{2}>0\right)=\frac{\pi}{4} \Theta\left(-R_{1}^{2} R_{2}^{2} R_{3}^{2}\right) .
$$

Note that since $\delta\left(x_{01}^{2}\right)$ is the Green's function of the d'Alembertian, see (11), the above integral $I_{3 \delta}$ satisfies

$$
\partial_{1}^{2} I_{3 \delta}=4 \pi \delta\left(x_{12}^{2}\right) \delta\left(x_{13}^{2}\right) .
$$

In the region $\sigma^{2}>0$ this is guaranteed by the Heaviside function in (A6); dropping the $\Theta$-function in (A6) would yield a vanishing result as $\partial_{1}^{2} \sigma^{-1}=0$.

\section{The case $\sigma^{2}<0$}

In the region where $\sigma^{2}<0$, we can span $x_{01}$ as

$$
x_{01}^{\mu}=t T^{\mu}+\tau R_{2}^{\mu}+\bar{\tau} R_{3}^{\mu}+r \xi^{\mu} .
$$

Here $T^{\mu}$ and $\xi^{\mu}$ denote again unit vectors that are orthogonal to each other and to $R_{2}^{\mu}, R_{3}^{\mu}$, with $T^{\mu}$ being timelike and $\xi^{\mu}$ spacelike. The volume element in this coordinate system is

$$
\mathrm{d}^{4} x_{0}=\sqrt{-\sigma^{2}} \mathrm{~d} t \mathrm{~d} r \mathrm{~d} \tau \mathrm{d} \bar{\tau}
$$

and the integral becomes

$$
\begin{aligned}
I_{3 \delta}= & \int \mathrm{d}^{4} x_{0} \delta\left(t^{2}+\left(\tau R_{2}^{\mu}+\bar{\tau} R_{3}^{\mu}\right)^{2}-r^{2}\right) \\
& \times \delta\left(2 \tau R_{2} \cdot R_{3}+(2 \bar{\tau}+1) R_{3}^{2}\right) \\
& \times \delta\left(2 \bar{\tau} R_{2} \cdot R_{3}+(2 \tau-1) R_{2}^{2}\right) \\
= & \frac{\sqrt{-\sigma^{2}}}{-4 \sigma^{2}} \int \mathrm{d} t \mathrm{~d} r \delta\left(t^{2}-r^{2}-\frac{R_{1}^{2} R_{2}^{2} R_{3}^{2}}{4 \sigma^{2}}\right) \\
= & \frac{1}{4 \sqrt{-\sigma^{2}}} \int_{-\infty}^{+\infty} \frac{\mathrm{d} r}{\sqrt{r^{2}+1}} \rightarrow \infty .
\end{aligned}
$$

Hence, for $\sigma^{2}<0$ the integral diverges.

\section{The case $\sigma^{2}=0$}

Finally, for $\sigma^{2}=0$ the surface spanned by the vectors connecting $x_{1}, x_{2}$ and $x_{3}$ degenerates into a line. We define the unit vector on this line as $R_{u}^{\mu}$, and we set $R_{i}^{\mu}=\omega_{i} R_{u}^{\mu}$. Depending on the nature of this line one finds different expressions as follows. For the line being timelike we have

$$
\begin{aligned}
x_{01}^{\mu} & =\tau R_{u}^{\mu}+r\left(\xi_{1}^{\mu} \cos \theta+\xi_{2}^{\mu} \sin \theta \cos \phi+\xi_{3}^{\mu} \sin \theta \sin \phi\right), \\
\mathrm{d}^{4} x_{0} & =r^{2} \sin \theta \mathrm{d} \tau \mathrm{d} r \mathrm{~d} \theta \mathrm{d} \phi,
\end{aligned}
$$

and thus

$$
\begin{aligned}
I_{3 \delta} & =\int \mathrm{d}^{4} x_{0} \delta\left(\tau^{2}-r^{2}\right) \delta\left(\omega_{3}^{2}+2 \tau \omega_{3}\right) \delta\left(\omega_{2}^{2}-2 \tau \omega_{2}\right) \\
& = \begin{cases}\infty & \omega_{1} \omega_{2} \omega_{3}=0 \\
0 & \text { otherwise }\end{cases}
\end{aligned}
$$

For a spacelike line and with $T \cdot R_{u}=0$, we have 


$$
\begin{aligned}
x_{01}^{\mu} & =t T^{\mu}+\tau R_{u}^{\mu}+r\left(\xi_{1}^{\mu} \cos \theta+\xi_{2}^{\mu} \sin \theta\right), \\
\mathrm{d}^{4} x_{0} & =r \mathrm{~d} t \mathrm{~d} \tau \mathrm{d} r \mathrm{~d} \theta,
\end{aligned}
$$

which implies

$$
\begin{aligned}
I_{3 \delta} & =\int \mathrm{d}^{4} x_{0} \delta\left(t^{2}+\tau^{2}-r^{2}\right) \delta\left(\omega_{3}^{2}+2 \tau \omega_{3}\right) \delta\left(\omega_{2}^{2}-2 \tau \omega_{2}\right) \\
& = \begin{cases}\infty & \omega_{1} \omega_{2} \omega_{3}=0, \\
0 & \text { otherwise }\end{cases}
\end{aligned}
$$

And finally for a lightlike line with $T \cdot R_{u} \neq 0$, we obtain

$$
\begin{aligned}
x_{01}^{\mu} & =t T^{\mu}+\tau R_{u}^{\mu}+r\left(\xi_{1}^{\mu} \cos \theta+\xi_{2}^{\mu} \sin \theta\right), \\
\mathrm{d}^{4} x_{0} & =\sqrt{\left(T \cdot R_{u}\right)^{2}-T^{2} R_{u}^{2}} r \mathrm{~d} t \mathrm{~d} \tau \mathrm{d} r \mathrm{~d} \theta,
\end{aligned}
$$

such that

$$
\begin{aligned}
I_{3 \delta}= & \int \mathrm{d}^{4} x_{0} \delta\left(t^{2}+2 t \tau T \cdot R_{u}-r^{2}\right) \\
& \times \delta\left(2 t \omega_{3} T \cdot R_{u}\right) \delta\left(2 t \omega_{2} T \cdot R_{u}\right) \\
& \sim \int \mathrm{d} \tau \delta(0)=\infty .
\end{aligned}
$$

Hence, the result for $\sigma^{2}=0$ may be summarized as

$$
I_{3 \delta}\left(\sigma^{2}=0\right) \sim \delta\left(R_{1}^{2}\right)+\delta\left(R_{2}^{2}\right)+\delta\left(R_{3}^{2}\right) .
$$

In total we thus conclude that the $3 \delta$-integral can be expressed as

$$
I_{3 \delta}= \begin{cases}\frac{\pi}{4 \sigma} \Theta\left(-R_{1}^{2} R_{2}^{2} R_{3}^{2}\right), & \sigma^{2}>0, \\ \sim \delta\left(R_{1}^{2}\right)+\delta\left(R_{2}^{2}\right)+\delta\left(R_{3}^{2}\right), & \sigma^{2}=0, \\ \infty, & \sigma^{2}<0 .\end{cases}
$$

We note that when using the result for $\sigma^{2}>0$ it can be useful to expand the theta-function according to

$$
\begin{aligned}
\Theta\left(-R_{1}^{2} R_{2}^{2} R_{3}^{2}\right)= & +\Theta\left(-R_{1}^{2}\right) \Theta\left(-R_{2}^{2}\right) \Theta\left(-R_{3}^{2}\right) \\
& +\Theta\left(-R_{1}^{2}\right) \Theta\left(+R_{2}^{2}\right) \Theta\left(+R_{3}^{2}\right) \\
& +\Theta\left(+R_{1}^{2}\right) \Theta\left(-R_{2}^{2}\right) \Theta\left(+R_{3}^{2}\right) \\
& +\Theta\left(+R_{1}^{2}\right) \Theta\left(+R_{2}^{2}\right) \Theta\left(-R_{3}^{2}\right) .
\end{aligned}
$$

\section{APPENDIX B: DERIVATIVES OF THE $3 \delta$ INTEGRAL}

In this Appendix we explicitly evaluate the expressions for the second order derivatives of the triple-delta integral $I_{3 \delta}$ for $\sigma^{2}>0$, cf. (A20). These enter into the three-body effective potential via (16). A priori we find four terms

$$
\begin{aligned}
\partial_{1}^{\mu} \partial_{2}^{\nu} I_{3 \delta}\left(\sigma^{2}>0\right)= & +\left(\partial_{1}^{\mu} \partial_{2}^{\nu} \frac{\pi}{4 \sigma}\right) \Theta\left(-R_{1}^{2} R_{2}^{2} R_{3}^{2}\right)+\left(\partial_{1}^{\mu} \frac{\pi}{4 \sigma}\right) \partial_{2}^{\nu} \Theta\left(-R_{1}^{2} R_{2}^{2} R_{3}^{2}\right) \\
& +\left(\partial_{2}^{\nu} \frac{\pi}{4 \sigma}\right) \partial_{1}^{\mu} \Theta\left(-R_{1}^{2} R_{2}^{2} R_{3}^{2}\right)+\frac{\pi}{4 \sigma} \partial_{1}^{\mu} \partial_{2}^{\nu} \Theta\left(-R_{1}^{2} R_{2}^{2} R_{3}^{2}\right),
\end{aligned}
$$

which evaluate to

$$
\begin{aligned}
\Theta\left(-R_{1}^{2} R_{2}^{2} R_{3}^{2}\right) \partial_{1}^{\mu} \partial_{2}^{\nu} \frac{\pi}{4 \sigma}= & \frac{\pi \Theta\left(-R_{1}^{2} R_{2}^{2} R_{3}^{2}\right)}{4 \sigma^{5}}\left[3\left(R_{1} \cdot R_{2}\right)\left(R_{1}^{\mu} R_{1}^{\nu} R_{2}^{2}+R_{2}^{\mu} R_{2}^{\nu} R_{1}^{2}-R_{1}^{\mu} R_{2}^{\nu} R_{1} \cdot R_{2}-R_{2}^{\mu} R_{1}^{\nu} R_{1} \cdot R_{2}\right)\right. \\
& \left.+\sigma^{2}\left(\eta^{\mu \nu} R_{1} \cdot R_{2}+R_{1}^{\mu} R_{2}^{\nu}+R_{2}^{\mu} R_{1}^{\nu}\right)\right], \\
\frac{\pi}{4 \sigma} \partial_{1}^{\mu} \partial_{2}^{\nu} \Theta\left(-R_{1}^{2} R_{2}^{2} R_{3}^{2}\right)= & \frac{\pi}{4 \sigma}\left[2 \eta^{\mu \nu} \delta\left(R_{3}^{2}\right)+4 R_{3}^{\mu} R_{3}^{\nu} \delta^{\prime}\left(R_{3}^{2}\right)\right] \operatorname{sgn}\left(R_{1}^{2} R_{2}^{2}\right)+\frac{8 \pi}{4 \sigma}\left[R_{2}^{\mu} R_{1}^{\nu} \delta\left(R_{1}^{2}\right) \delta\left(R_{2}^{2}\right) \operatorname{sgn}\left(R_{3}^{2}\right)\right. \\
& \left.-R_{2}^{\mu} R_{3}^{\nu} \delta\left(R_{2}^{2}\right) \delta\left(R_{3}^{2}\right) \operatorname{sgn}\left(R_{1}^{2}\right)-R_{3}^{\mu} R_{1}^{\nu} \delta\left(R_{1}^{2}\right) \delta\left(R_{3}^{2}\right) \operatorname{sgn}\left(R_{2}^{2}\right)\right], \\
\left(\partial_{1}^{\mu} \frac{\pi}{4 \sigma}\right) \partial_{2}^{\nu} \Theta\left(-R_{1}^{2} R_{2}^{2} R_{3}^{2}\right)= & \frac{\pi}{4 \sigma}\left[\frac{4 R_{1}^{\mu} R_{1}^{\nu}}{R_{2}^{2}-R_{3}^{2}} \delta\left(R_{1}^{2}\right) \operatorname{sgn}\left(R_{2}^{2} R_{3}^{2}\right)\right. \\
& \left.-\frac{4 R_{2}^{\mu} R_{3}^{\nu}}{R_{1}^{2}-R_{2}^{2}} \delta\left(R_{3}^{2}\right) \operatorname{sgn}\left(R_{1}^{2} R_{2}^{2}\right)+R_{3}^{\mu} R_{3}^{\nu} \frac{R_{1}^{2}+R_{2}^{2}}{\sigma^{2}} \delta\left(R_{3}^{2}\right) \operatorname{sgn}\left(R_{1}^{2} R_{2}^{2}\right)\right] .
\end{aligned}
$$

Here the last line also enters into (B1) with the labels 1 and 2 interchanged. Note the appearance of the derivative of the delta function in the first line of (B3) that one could resolve using $\delta^{\prime}\left(R_{3}^{2}\right)=-\delta\left(R_{3}^{2}\right) / R_{3}^{2}$. Putting these terms together, Eq. (B1) then becomes (ordered by the number of delta functions) 


$$
\begin{aligned}
\partial_{1}^{\mu} \partial_{2}^{\nu} I_{3 \delta}= & \frac{\pi \Theta\left(-R_{1}^{2} R_{2}^{2} R_{3}^{2}\right)}{4 \sigma^{5}}\left[3\left(R_{1} \cdot R_{2}\right)\left(R_{1}^{\mu} R_{1}^{\nu} R_{2}^{2}+R_{2}^{\mu} R_{2}^{\nu} R_{1}^{2}-R_{1}^{\mu} R_{2}^{\nu} R_{1} \cdot R_{2}-R_{2}^{\mu} R_{1}^{\nu} R_{1} \cdot R_{2}\right)+\sigma^{2}\left(\eta^{\mu \nu} R_{1} \cdot R_{2}+R_{1}^{\mu} R_{2}^{\nu}+R_{2}^{\mu} R_{1}^{\nu}\right)\right] \\
& +\frac{\pi}{4 \sigma}\left[+\frac{4 R_{1}^{\mu} R_{1}^{\nu}}{R_{2}^{2}-R_{3}^{2}} \delta\left(R_{1}^{2}\right) \operatorname{sgn}\left(R_{2}^{2} R_{3}^{2}\right)+\frac{4 R_{2}^{\mu} R_{2}^{\nu}}{R_{1}^{2}-R_{3}^{2}} \delta\left(R_{2}^{2}\right) \operatorname{sgn}\left(R_{1}^{2} R_{3}^{2}\right)\right] \\
& +\frac{\pi}{4 \sigma}\left[-\frac{4 R_{1}^{\mu} R_{3}^{\nu}}{R_{2}^{2}-R_{1}^{2}} \delta\left(R_{3}^{2}\right)-\frac{4 R_{2}^{\mu} R_{3}^{\nu}}{R_{1}^{2}-R_{2}^{2}} \delta\left(R_{3}^{2}\right)+2 R_{3}^{\mu} R_{3}^{\nu} \frac{R_{1}^{2}+R_{2}^{2}}{\sigma^{2}} \delta\left(R_{3}^{2}\right)+2 \eta^{\mu \nu} \delta\left(R_{3}^{2}\right)+4 R_{3}^{\mu} R_{3}^{\nu} \delta^{\prime}\left(R_{3}^{2}\right)\right] \operatorname{sgn}\left(R_{1}^{2} R_{2}^{2}\right) \\
& +\frac{8 \pi}{4 \sigma}\left[+R_{2}^{\mu} R_{1}^{\nu} \delta\left(R_{1}^{2}\right) \delta\left(R_{2}^{2}\right) \operatorname{sgn}\left(R_{3}^{2}\right)-R_{2}^{\mu} R_{3}^{\nu} \delta\left(R_{2}^{2}\right) \delta\left(R_{3}^{2}\right) \operatorname{sgn}\left(R_{1}^{2}\right)-R_{3}^{\mu} R_{1}^{\nu} \delta\left(R_{1}^{2}\right) \delta\left(R_{3}^{2}\right) \operatorname{sgn}\left(R_{2}^{2}\right)\right] .
\end{aligned}
$$

Performing the PN expansion starting from this expression seems (also conceptually) much harder than working on the level of the integrand of $I_{3 \delta}$ in (16). The latter is demonstrated in Sec. V.

\section{APPENDIX C: DETAILS ON 3PN}

In the computation of the $3 \mathrm{PN}$ potential, we added the following total derivative to remove the dependence on the derivative of accelerations and possible spurious poles for $r_{i j} \rightarrow \infty$ :

$$
L^{\mathrm{td}}=\sum_{j \neq i} \frac{G m_{i} m_{j}}{48 c^{8}} \frac{\mathrm{d}}{\mathrm{d} t}\left[r_{i j}\left(21 \mathbf{a}_{i} \cdot \mathbf{v}_{j}-18 \mathbf{a}_{i} \cdot \mathbf{v}_{i}\right)\left(\left(\mathbf{n}_{i j} \cdot \mathbf{v}_{j}\right)^{2}+\mathbf{v}_{j}^{2}\right)+r_{i j} \mathbf{n}_{i j} \cdot \mathbf{a}_{i} \mathbf{n}_{i j} \cdot \mathbf{v}_{j}\left(\left(\mathbf{n}_{i j} \cdot \mathbf{v}_{j}\right)^{2}-3 \mathbf{v}_{j}^{2}\right)\right]
$$

Due to its length, here we display only an excerpt of the genuine three-body contribution to the 3PN effective potential from the third line of (16). The full result is given in an ancillary file [81]. The expression below is organized according to the rational functions of the spatial distances, where each function is multiplied by a sum of numerator structures that scale as $v^{4}$. Note that some numerator structures begin with the same terms but they do not agree. Evaluating the derivatives in (57) yields the expression

$$
\begin{aligned}
L_{(D)}^{3 \text { PN }}= & \sum_{j \neq i} \sum_{k \neq i, j} G^{2} m_{i} m_{j} m_{k} \\
& \times\left\{\frac{1}{\left(r_{i j}+r_{j k}+r_{i k}\right)^{2}}\left(\left(\mathbf{n}_{i k} \cdot \mathbf{v}_{i}\right)\left(\frac{16}{3}\left(\mathbf{n}_{i j} \cdot \mathbf{v}_{i}\right)^{3}-12\left(\mathbf{n}_{i j} \cdot \mathbf{v}_{i}\right)^{2}\left(\mathbf{n}_{i j} \cdot \mathbf{v}_{j}\right)+\frac{20}{3}\left(\mathbf{n}_{i j} \cdot \mathbf{v}_{i}\right)\left(\mathbf{n}_{i j} \cdot \mathbf{v}_{j}\right)^{2}\right)+245 \text { terms }\right)\right. \\
& +\frac{1}{r_{i j}\left(r_{i j}+r_{j k}+r_{i k}\right)}\left(\mathbf{v}_{i}^{2}\left(\frac{16}{3}\left(\mathbf{n}_{i j} \cdot \mathbf{v}_{i}\right)^{2}-11\left(\mathbf{n}_{i j} \cdot \mathbf{v}_{i}\right)\left(\mathbf{n}_{i j} \cdot \mathbf{v}_{j}\right)+\frac{16}{3}\left(\mathbf{n}_{i j} \cdot \mathbf{v}_{j}\right)^{2}\right)+45 \text { terms }\right) \\
& -\frac{r_{i j}}{\left(r_{i j}+r_{j k}+r_{i k}\right)^{3}}\left(\frac{8}{3}\left(\mathbf{n}_{i j} \cdot \mathbf{v}_{i}\right)^{4}-6\left(\mathbf{n}_{i j} \cdot \mathbf{v}_{i}\right)^{3}\left(\mathbf{n}_{i j} \cdot \mathbf{v}_{j}\right)+\frac{8}{3}\left(\mathbf{n}_{i j} \cdot \mathbf{v}_{i}\right)^{2}\left(\mathbf{n}_{i j} \cdot \mathbf{v}_{j}\right)^{2}+286 \text { terms }\right) \\
& -\frac{r_{i k} r_{j k}}{r_{i j}\left(r_{i j}+r_{j k}+r_{i k}\right)^{3}}\left(\left(\mathbf{n}_{i k} \cdot \mathbf{v}_{k}\right)^{2}\left(16\left(\mathbf{n}_{i j} \cdot \mathbf{v}_{i}\right)^{2}-36\left(\mathbf{n}_{i j} \cdot \mathbf{v}_{i}\right)\left(\mathbf{n}_{i j} \cdot \mathbf{v}_{j}\right)+16\left(\mathbf{n}_{i j} \cdot \mathbf{v}_{j}\right)^{2}\right)+69 \text { terms }\right) \\
& -\frac{r_{i j}^{2}}{\left(r_{i j}+r_{j k}+r_{i k}\right)^{4}}\left(8\left(\mathbf{n}_{i j} \cdot \mathbf{v}_{i}\right)^{4}-18\left(\mathbf{n}_{i j} \cdot \mathbf{v}_{i}\right)^{3}\left(\mathbf{n}_{i j} \cdot \mathbf{v}_{j}\right)+16\left(\mathbf{n}_{i j} \cdot \mathbf{v}_{i}\right)^{2}\left(\mathbf{n}_{i j} \cdot \mathbf{v}_{j}\right)^{2}+143 \text { terms }\right) \\
& +\frac{r_{i k} r_{j k}}{\left(r_{i j}+r_{j k}+r_{i k}\right)^{4}}\left(\left(\mathbf{n}_{i k} \cdot \mathbf{v}_{k}\right)^{2}\left(16\left(\mathbf{n}_{i j} \cdot \mathbf{v}_{i}\right)^{2}-36\left(\mathbf{n}_{i j} \cdot \mathbf{v}_{i}\right)\left(\mathbf{n}_{i j} \cdot \mathbf{v}_{j}\right)+16\left(\mathbf{n}_{i j} \cdot \mathbf{v}_{j}\right)^{2}\right)+114 \text { terms }\right) \\
& -\left[\frac{1}{r_{i k}\left(r_{i j}+r_{j k}+r_{i k}\right)}\left(\frac{4}{3} \mathbf{v}_{i}^{4}-\frac{4}{3}\left(\left(\mathbf{n}_{i k} \cdot \mathbf{v}_{i}\right)^{2}-\left(\mathbf{n}_{i k} \cdot \mathbf{v}_{k}\right)^{2}\right) \mathbf{v}_{i}^{2}+20 \text { terms }\right)+(i \leftrightarrow j)\right] \\
& -\left[\frac{r_{i k}}{r_{j k}\left(r_{i j}+r_{j k}+r_{i k}\right)^{2}}\left(\left(\mathbf{n}_{j k} \cdot \mathbf{v}_{k}\right)^{2}\left(\frac{8}{3}\left(\mathbf{n}_{i j} \cdot \mathbf{v}_{i}\right)^{2}-6\left(\mathbf{n}_{i j} \cdot \mathbf{v}_{i}\right)\left(\mathbf{n}_{i j} \cdot \mathbf{v}_{j}\right)+\frac{8}{3}\left(\mathbf{n}_{i j} \cdot \mathbf{v}_{j}\right)^{2}\right)+23 \text { terms }\right)+(i \leftrightarrow j)\right] \\
& +\left[\frac{r_{i j}}{r_{i k}\left(r_{i j}+r_{j k}+r_{i k}\right)^{2}}\left(\left(\mathbf{n}_{j k} \cdot \mathbf{v}_{k}\right)^{2}\left(\frac{8}{3}\left(\mathbf{n}_{i j} \cdot \mathbf{v}_{i}\right)^{2}-6\left(\mathbf{n}_{i j} \cdot \mathbf{v}_{i}\right)\left(\mathbf{n}_{i j} \cdot \mathbf{v}_{j}\right)+\frac{8}{3}\left(\mathbf{n}_{i j} \cdot \mathbf{v}_{j}\right)^{2}\right)+47 \text { terms }\right)+(i \leftrightarrow j)\right]
\end{aligned}
$$




$$
\begin{aligned}
& -\left[\frac{r_{i k}}{r_{i j}\left(r_{i j}+r_{j k}+r_{i k}\right)^{2}}\left(8\left(\mathbf{n}_{i j} \cdot \mathbf{v}_{i}\right)^{4}-18\left(\mathbf{n}_{i j} \cdot \mathbf{v}_{i}\right)^{3}\left(\mathbf{n}_{i j} \cdot \mathbf{v}_{j}\right)+12\left(\mathbf{n}_{i j} \cdot \mathbf{v}_{i}\right)^{2}\left(\mathbf{n}_{i j} \cdot \mathbf{v}_{j}\right)^{2}+93 \text { terms }\right)+(i \leftrightarrow j)\right] \\
& -\left[\frac{r_{i k}}{\left(r_{i j}+r_{j k}+r_{i k}\right)^{3}}\left(16\left(\mathbf{n}_{i j} \cdot \mathbf{v}_{i}\right)^{4}-36\left(\mathbf{n}_{i j} \cdot \mathbf{v}_{i}\right)^{3}\left(\mathbf{n}_{i j} \cdot \mathbf{v}_{j}\right)+24\left(\mathbf{n}_{i j} \cdot \mathbf{v}_{i}\right)^{2}\left(\mathbf{n}_{i j} \cdot \mathbf{v}_{j}\right)^{2}+285 \text { terms }\right)+(i \leftrightarrow j)\right] \\
& +\left[\frac{r_{i k}^{2}}{r_{j k}\left(r_{i j}+r_{j k}+r_{i k}\right)^{3}}\left(\left(\mathbf{n}_{j k} \cdot \mathbf{v}_{k}\right)^{2}\left(\frac{16}{3}\left(\mathbf{n}_{i j} \cdot \mathbf{v}_{i}\right)^{2}-12\left(\mathbf{n}_{i j} \cdot \mathbf{v}_{i}\right)\left(\mathbf{n}_{i j} \cdot \mathbf{v}_{j}\right)+\frac{16}{3}\left(\mathbf{n}_{i j} \cdot \mathbf{v}_{j}\right)^{2}\right)+46 \text { terms }\right)+(i \leftrightarrow j)\right] \\
& -\left[\frac{r_{i j}^{2}}{r_{i k}\left(r_{i j}+r_{j k}+r_{i k}\right)^{3}}\left(\left(\mathbf{n}_{i k} \cdot \mathbf{v}_{i}\right)^{2}\left(\frac{8}{3}\left(\mathbf{n}_{i j} \cdot \mathbf{v}_{i}\right)^{2}-6\left(\mathbf{n}_{i j} \cdot \mathbf{v}_{i}\right)\left(\mathbf{n}_{i j} \cdot \mathbf{v}_{j}\right)+\frac{8}{3}\left(\mathbf{n}_{i j} \cdot \mathbf{v}_{j}\right)^{2}\right)+58 \text { terms }\right)+(i \leftrightarrow j)\right] \\
& -\left[\frac{r_{i j} r_{j k}}{r_{i k}\left(r_{i j}+r_{j k}+r_{i k}\right)^{3}}\left(\left(\mathbf{n}_{i k} \cdot \mathbf{v}_{i}\right)^{2}\left(\frac{8}{3}\left(\mathbf{n}_{i j} \cdot \mathbf{v}_{i}\right)^{2}-6\left(\mathbf{n}_{i j} \cdot \mathbf{v}_{i}\right)\left(\mathbf{n}_{i j} \cdot \mathbf{v}_{j}\right)+\frac{8}{3}\left(\mathbf{n}_{i j} \cdot \mathbf{v}_{j}\right)^{2}\right)+72 \text { terms }\right)+(i \leftrightarrow j)\right] \\
& -\left[\frac{r_{i k}^{2}}{r_{i j}\left(r_{i j}+r_{j k}+r_{i k}\right)^{3}}\left(\left(\mathbf{n}_{i k} \cdot \mathbf{v}_{k}\right)^{2}\left(\frac{40}{3}\left(\mathbf{n}_{i k} \cdot \mathbf{v}_{i}\right)^{2}-30\left(\mathbf{n}_{i k} \cdot \mathbf{v}_{i}\right)\left(\mathbf{n}_{i k} \cdot \mathbf{v}_{j}\right)+\frac{40}{3}\left(\mathbf{n}_{i k} \cdot \mathbf{v}_{j}\right)^{2}\right)+75 \text { terms }\right)+(i \leftrightarrow j)\right] \\
& +\left[\frac{r_{i k}^{2}}{\left(r_{i j}+r_{j k}+r_{i k}\right)^{4}}\left(\left(\mathbf{n}_{i k} \cdot \mathbf{v}_{k}\right)^{2}\left(16\left(\mathbf{n}_{i j} \cdot \mathbf{v}_{i}\right)^{2}-36\left(\mathbf{n}_{i j} \cdot \mathbf{v}_{i}\right)\left(\mathbf{n}_{i j} \cdot \mathbf{v}_{j}\right)+16\left(\mathbf{n}_{i j} \cdot \mathbf{v}_{j}\right)^{2}\right)+109 \text { terms }\right)+(i \leftrightarrow j)\right] \\
& -\left[\frac{r_{i j} r_{i k}}{\left(r_{i j}+r_{j k}+r_{i k}\right)^{4}}\left(16\left(\mathbf{n}_{i j} \cdot \mathbf{v}_{i}\right)^{4}-36\left(\mathbf{n}_{i j} \cdot \mathbf{v}_{i}\right)^{3}\left(\mathbf{n}_{i j} \cdot \mathbf{v}_{j}\right)+24\left(\mathbf{n}_{i j} \cdot \mathbf{v}_{i}\right)^{2}\left(\mathbf{n}_{i j} \cdot \mathbf{v}_{j}\right)^{2}+174 \text { terms }\right)+(i \leftrightarrow j)\right] \\
& -\left[\frac{r_{i k}^{2}\left(r_{i j}+r_{j k}\right)}{r_{i j} r_{j k}\left(r_{i j}+r_{j k}+r_{i k}\right)^{3}}\left(\left(\mathbf{n}_{j k} \cdot \mathbf{v}_{k}\right)^{2}\left(\frac{8}{3}\left(\mathbf{n}_{i j} \cdot \mathbf{v}_{i}\right)^{2}-6\left(\mathbf{n}_{i j} \cdot \mathbf{v}_{i}\right)\left(\mathbf{n}_{i j} \cdot \mathbf{v}_{j}\right)+\frac{8}{3}\left(\mathbf{n}_{i j} \cdot \mathbf{v}_{j}\right)^{2}\right)+21 \text { terms }\right)+(i \leftrightarrow j)\right] \\
& \left.+\left[\frac{r_{i k}^{2}\left(r_{i k}+2 r_{j k}\right)}{r_{i j} r_{j k}\left(r_{i j}+r_{j k}+r_{i k}\right)^{4}}\left(\left(\mathbf{n}_{i k} \cdot \mathbf{v}_{k}\right)^{2}\left(8\left(\mathbf{n}_{i j} \cdot \mathbf{v}_{i}\right)^{2}-36\left(\mathbf{n}_{i j} \cdot \mathbf{v}_{i}\right)\left(\mathbf{n}_{i j} \cdot \mathbf{v}_{j}\right)+8\left(\mathbf{n}_{i j} \cdot \mathbf{v}_{j}\right)^{2}\right)+33 \text { terms }\right)+(i \leftrightarrow j)\right]\right\} .
\end{aligned}
$$

[1] H. Poincaré, Sur le problème des trois corps et les équations de la dynamique, Acta Math. 13, 1 (1890).

[2] C. Liu, The Three-Body Problem (Tor Books, New York, 2007).

[3] C. M. Will, Incorporating post-Newtonian effects in $N$-body dynamics, Phys. Rev. D 89, 044043 (2014); , Erratum, 91, 029902 (2015).

[4] B. P. Abbott et al. (LIGO Scientific and Virgo Collaborations), Observation of Gravitational Waves from a Binary Black Hole Merger, Phys. Rev. Lett. 116, 061102 (2016).

[5] B. P. Abbott et al. (LIGO Scientific and Virgo Collaborations), GW170817: Observation of Gravitational Waves from a Binary Neutron Star Inspiral, Phys. Rev. Lett. 119, 161101 (2017).

[6] B. P. Abbott et al. (LIGO Scientific and Virgo Collaborations), GWTC-1: A Gravitational-Wave Transient Catalog of Compact Binary Mergers Observed by LIGO and Virgo during the First and Second Observing Runs, Phys. Rev. X 9, 031040 (2019).

[7] H. Asada, Gravitational wave forms for a three-body system in Lagrange's orbit: Parameter determinations and a binary source test, Phys. Rev. D 80, 064021 (2009).
[8] P. Galaviz and B. Bruegmann, Characterization of the gravitational wave emission of three black holes, Phys. Rev. D 83, 084013 (2011).

[9] Y. Meiron, B. Kocsis, and A. Loeb, Detecting triple systems with gravitational wave observations, Astrophys. J. 834, 200 (2017).

[10] M. Bonetti, E. Barausse, G. Faye, F. Haardt, and A. Sesana, About gravitational-wave generation by a three-body system, Classical Quantum Gravity 34, 215004 (2017).

[11] H. Lim and C. L. Rodriguez, Relativistic three-body effects in hierarchical triples, Phys. Rev. D 102, 064033 (2020).

[12] A. Einstein, L. Infeld, and B. Hoffmann, The gravitational equations and the problem of motion, Ann. Math. 39, 65 (1938).

[13] A. S. Eddington and G. L. Clark, The problem of $\mathrm{n}$ bodies in general relativity theory, Proc. R. Soc. A 166, 465 (1938).

[14] L. D. Landau, Textbook on Theoretical Physics. Vol. 2: Classical Field Theory, edited by E. M. Lifshitz, H. G. Schopf, and P. Ziesche (Akademie Verlag, Berlin, 1987) (in German). 
[15] K. Gultekin, M. C. Miller, and D. P. Hamilton, Three-body dynamics with gravitational wave emission, Astrophys. J. 640, 156 (2006).

[16] M. Iwasawa, Y. Funato, and J. Makino, Evolution of massive blackhole triples. 1. Equal-mass binary-single systems, Astrophys. J. 651, 1059 (2006).

[17] L. Hoffman and A. Loeb, Dynamics of triple black hole systems in hierarchically merging massive galaxies, Mon. Not. R. Astron. Soc. 377, 957 (2007).

[18] P. Gupta, H. Suzuki, H. Okawa, and K.-i. Maeda, Gravitational waves from hierarchical triple systems with KozaiLidov oscillation, Phys. Rev. D 101, 104053 (2020).

[19] C. O. Lousto and H. Nakano, Three-body equations of motion in successive post-Newtonian approximations, Classical Quantum Gravity 25, 195019 (2008).

[20] P. Galaviz, Stability and chaos of hierarchical three black hole configurations, Phys. Rev. D 84, 104038 (2011).

[21] S. Naoz, B. Kocsis, A. Loeb, and N. Yunes, Resonant postNewtonian eccentricity excitation in hierarchical three-body systems, Astrophys. J. 773, 187 (2013).

[22] M. Bonetti, F. Haardt, A. Sesana, and E. Barausse, PostNewtonian evolution of massive black hole triplets in galactic nuclei-I. Numerical implementation and tests, Mon. Not. R. Astron. Soc. 461, 4419 (2016).

[23] M. Campanelli, C. O. Lousto, and Y. Zlochower, Close encounters of three black holes, Phys. Rev. D 77, 101501 (2008).

[24] C. O. Lousto and Y. Zlochower, Foundations of multiple black hole evolutions, Phys. Rev. D 77, 024034 (2008).

[25] P. Galaviz, B. Bruegmann, and Z. Cao, Numerical evolution of multiple black holes with accurate initial data, Phys. Rev. D 82, 024005 (2010).

[26] T. Damour, P. Jaranowski, and G. Schäfer, Nonlocal-intime action for the fourth post-Newtonian conservative dynamics of two-body systems, Phys. Rev. D 89, 064058 (2014).

[27] L. Bernard, L. Blanchet, A. Bohé, G. Faye, and S. Marsat, Fokker action of nonspinning compact binaries at the fourth post-Newtonian approximation, Phys. Rev. D 93, 084037 (2016).

[28] T. Damour, P. Jaranowski, and G. Schäfer, Conservative dynamics of two-body systems at the fourth post-Newtonian approximation of general relativity, Phys. Rev. D 93, 084014 (2016).

[29] L. Bernard, L. Blanchet, A. Bohé, G. Faye, and S. Marsat, Energy and periastron advance of compact binaries on circular orbits at the fourth post-Newtonian order, Phys. Rev. D 95, 044026 (2017).

[30] S. Foffa, P. Mastrolia, R. Sturani, and C. Sturm, Effective field theory approach to the gravitational two-body dynamics, at fourth post-Newtonian order and quintic in the Newton constant, Phys. Rev. D 95, 104009 (2017).

[31] R. A. Porto and I. Z. Rothstein, Apparent ambiguities in the post-Newtonian expansion for binary systems, Phys. Rev. D 96, 024062 (2017).

[32] T. Marchand, L. Bernard, L. Blanchet, and G. Faye, Ambiguity-free completion of the equations of motion of compact binary systems at the fourth post-Newtonian order, Phys. Rev. D 97, 044023 (2018).
[33] T. Damour and P. Jaranowski, Four-loop static contribution to the gravitational interaction potential of two point masses, Phys. Rev. D 95, 084005 (2017).

[34] S. Foffa and R. Sturani, Conservative dynamics of binary systems to fourth post-Newtonian order in the EFT approach I: Regularized Lagrangian, Phys. Rev. D 100, 024047 (2019).

[35] S. Foffa, R. A. Porto, I. Rothstein, and R. Sturani, Conservative dynamics of binary systems to fourth PostNewtonian order in the EFT approach II: Renormalized Lagrangian, Phys. Rev. D 100, 024048 (2019).

[36] J. Blümlein, A. Maier, P. Marquard, and G. Schäfer, Fourth post-Newtonian Hamiltonian dynamics of two-body systems from an effective field theory approach, Nucl. Phys. B955, 115041 (2020).

[37] C. R. Galley, A. K. Leibovich, R. A. Porto, and A. Ross, Tail effect in gravitational radiation reaction: Time nonlocality and renormalization group evolution, Phys. Rev. D 93, 124010 (2016).

[38] S. Foffa, P. Mastrolia, R. Sturani, C. Sturm, and W. J. Torres Bobadilla, Static Two-Body Potential at Fifth Post-Newtonian Order, Phys. Rev. Lett. 122, 241605 (2019).

[39] J. Blümlein, A. Maier, and P. Marquard, Five-loop static contribution to the gravitational interaction potential of two point masses, Phys. Lett. B 800, 135100 (2020).

[40] D. Bini, T. Damour, and A. Geralico, Novel Approach to Binary Dynamics: Application to the Fifth Post-Newtonian Level, Phys. Rev. Lett. 123, 231104 (2019).

[41] J. Blümlein, A. Maier, P. Marquard, and G. Schäfer, Testing binary dynamics in gravity at the sixth post-Newtonian level, Phys. Lett. B 807, 135496 (2020).

[42] C. Cheung and M. P. Solon, Classical gravitational scattering at $\mathcal{O}\left(\mathrm{G}^{3}\right)$ from Feynman diagrams, J. High Energy Phys. 06 (2020) 144.

[43] D. Bini, T. Damour, and A. Geralico, Sixth post-Newtonian local-in-time dynamics of binary systems, Phys. Rev. D 102, 024061 (2020).

[44] D. Bini, T. Damour, and A. Geralico, Binary dynamics at the fifth and fifth-and-a-half post-Newtonian orders, Phys. Rev. D 102, 024062 (2020).

[45] D. Bini, T. Damour, and A. Geralico, Sixth post-Newtonian nonlocal-in-time dynamics of binary systems, Phys. Rev. D 102, 084047 (2020).

[46] D. Bini, T. Damour, A. Geralico, S. Laporta, and P. Mastrolia, Gravitational dynamics at $O\left(G^{6}\right)$ : perturbative gravitational scattering meets experimental mathematics, arXiv:2008.09389.

[47] T. Ohta, H. Okamura, K. Hiida, and T. Kimura, Higher order gravitational potential for many-body system, Prog. Theor. Phys. 51, 1220 (1974).

[48] T. Damour and G. Schäfer, Lagrangians from point masses at the second post-Newtonian approximation of general relativity, Gen. Relativ. Gravit. 17, 879 (1985).

[49] G. Schäfer, Three-body hamiltonian in general relativity, Phys. Lett. A 123, 336 (1987).

[50] Y.-Z. Chu, The n-body problem in general relativity up to the second post-Newtonian order from perturbative field theory, Phys. Rev. D 79, 044031 (2009). 
[51] C. Cheung, I. Z. Rothstein, and M. P. Solon, From Scattering Amplitudes to Classical Potentials in the Post-Minkowskian Expansion, Phys. Rev. Lett. 121, 251101 (2018).

[52] A. Cristofoli, N. E. J. Bjerrum-Bohr, P. H. Damgaard, and P. Vanhove, post-Minkowskian Hamiltonians in general relativity, Phys. Rev. D 100, 084040 (2019).

[53] Z. Bern, C. Cheung, R. Roiban, C.-H. Shen, M. P. Solon, and M. Zeng, Scattering Amplitudes and the Conservative Hamiltonian for Binary Systems at Third Post-Minkowskian Order, Phys. Rev. Lett. 122, 201603 (2019).

[54] Z. Bern, C. Cheung, R. Roiban, C.-H. Shen, M. P. Solon, and $\mathrm{M}$. Zeng, Black hole binary dynamics from the double copy and effective theory, J. High Energy Phys. 10 (2019) 206.

[55] T. Damour, Radiative contribution to classical gravitational scattering at the third order in $G$, Phys. Rev. D 102, 124008 (2020).

[56] P. Di Vecchia, C. Heissenberg, R. Russo, and G. Veneziano, Universality of ultra-relativistic gravitational scattering, Phys. Lett. B 811, 135924 (2020).

[57] G. Kälin and R. A. Porto, Post-Minkowskian effective field theory for conservative binary dynamics, J. High Energy Phys. 11 (2020) 106.

[58] G. Kälin, Z. Liu, and R. A. Porto, Conservative Dynamics of Binary Systems to Third Post-Minkowskian Order from the Effective Field Theory Approach, Phys. Rev. Lett. 125, 261103 (2020).

[59] K. Westpfahl, High-speed scattering of charged and uncharged particles in general relativity, Fortschr. Phys. 33, 417 (1985).

[60] L. Bel, T. Damour, N. Deruelle, J. Ibanez, and J. Martin, Poincaré-invariant gravitational field and equations of motion of two pointlike objects: The postlinear approximation of general relativity, Gen. Relativ. Gravit. 13, 963 (1981).

[61] T. Ledvinka, G. Schaefer, and J. Bicak, Relativistic ClosedForm Hamiltonian for Many-Body Gravitating Systems in the Post-Minkowskian Approximation, Phys. Rev. Lett. 100, 251101 (2008).

[62] T. Damour, Gravitational scattering, post-Minkowskian approximation and effective one-body theory, Phys. Rev. D 94, 104015 (2016).

[63] L. Blanchet and A. S. Fokas, Equations of motion of selfgravitating $N$-body systems in the first post-Minkowskian approximation, Phys. Rev. D 98, 084005 (2018).

[64] G. Mogull, J. Plefka, and J. Steinhoff, Classical black hole scattering from a worldline quantum field theory, J. High Energy Phys. 02 (2021) 048.

[65] W. D. Goldberger and I.Z. Rothstein, An effective field theory of gravity for extended objects, Phys. Rev. D 73, 104029 (2006).
[66] D. Chicherin, V. Kazakov, F. Loebbert, D. Müller, and D.-1. Zhong, Yangian symmetry for bi-scalar loop amplitudes, J. High Energy Phys. 05 (2018) 003.

[67] F. Loebbert, J. Miczajka, D. Müller, and H. Münkler, Massive Conformal Symmetry and Integrability for Feynman Integrals, Phys. Rev. Lett. 125, 091602 (2020).

[68] F. Loebbert, D. Müller, and H. Münkler, Yangian bootstrap for conformal Feynman integrals, Phys. Rev. D 101, 066006 (2020).

[69] L. Corcoran, F. Loebbert, J. Miczajka, and M. Staudacher, Minkowski box from Yangian bootstrap, arXiv:2012 .07852 .

[70] C. Coriano, L. Delle Rose, E. Mottola, and M. Serino, Solving the conformal constraints for scalar operators in momentum space and the evaluation of Feynman's master integrals, J. High Energy Phys. 07 (2013) 011.

[71] A. Bzowski, P. McFadden, and K. Skenderis, Implications of conformal invariance in momentum space, J. High Energy Phys. 03 (2014) 111.

[72] F. Loebbert, J. Miczajka, D. Müller, and H. Münkler, Yangian bootstrap for massive Feynman integrals, arXiv: 2010.08552.

[73] J. W. York, Jr., Role of Conformal Three Geometry in the Dynamics of Gravitation, Phys. Rev. Lett. 28, 1082 (1972).

[74] G. W. Gibbons and S. W. Hawking, Action integrals and partition functions in quantum gravity, Phys. Rev. D 15, 2752 (1977).

[75] S. Sannan, Gravity as the limit of the type $\{$ II $\}$ superstring theory, Phys. Rev. D 34, 1749 (1986).

[76] T. Damour and G. Schaefer, Redefinition of position variables and the reduction of higher order Lagrangians, J. Math. Phys. (N.Y.) 32, 127 (1991).

[77] D. Chicherin, V. Kazakov, F. Loebbert, D. Müller, and D.-1. Zhong, Yangian symmetry for fishnet Feynman graphs, Phys. Rev. D 96, 121901 (2017).

[78] E. E. Boos and A. I. Davydychev, A method of evaluating massive Feynman integrals, Theor. Math. Phys. 89, 1052 (1991).

[79] T. Ohta, H. Okamura, T. Kimura, and K. Hiida, Physically acceptable solution of Einstein's equation for many-body system, Prog. Theor. Phys. 50, 492 (1973).

[80] A. P. Isaev, Operator approach to analytical evaluation of Feynman diagrams, Phys. At. Nucl. 71, 914 (2008).

[81] See Supplemental Material at http://link.aps.org/ supplemental/10.1103/PhysRevD.103.064010 for ancillary file ExplicitExpressionsAt3PN.m including expressions for $L_{(A, B, C, D)}^{3 \mathrm{PN}}$ discussed in Sec. VII.

[82] F. Loebbert, M. Mojaza, and J. Plefka, Hidden conformal symmetry in tree-level graviton scattering, J. High Energy Phys. 05 (2018) 208. 